\title{
Classical p-adic Nevanlinna theory and Nevalinna Theory out of a hole
}

\author{
Alain Escassut and Ta Thi Hoai An
}

\begin{abstract}
In the first section called Classical theory, we recall basic properties of the analytic and meromorphic functions, Motzkin's factoraization of analytic elements and the classical p-adic Nevanlinna theory. The second section is devoted to meromorphic functions in the complement of an "open" disk with the use of Motzkin's factorization and we show the existence of a Nevanlinna theory in that field of meromorphic functions. Applications are examined: Nevanlinna theory on 3 small functions, parametrization of algebraic curves, qusai-exceptional small functions and branched small functions.
\end{abstract}

\section{Classical theory}

\section{I.1 Basic definitions}

We denote by $\mathbb{K}$ an algebraically closed field of characteristic 0 , complete with respect to an ultrametric absolute value $|$.$| . Analytic functions inside a disk or in$ the whole field $\mathbb{K}$ were introduced and studied in many books. Given $\alpha \in \mathbb{K}$ and $R \in \mathbb{R}_{+}^{*}$, we denote by $d(\alpha, R)$ the disk $\{x \in \mathbb{K}|| x-\alpha \mid \leq R\}$, by $d\left(\alpha, R^{-}\right)$the disk $\{x \in \mathbb{K}|| x-\alpha \mid<R\}$ and by $C(\alpha, r)$ the circle $\{x \in \mathbb{K}|| x-\alpha \mid=r\}$. We denote by $|\mathbb{K}|$ the set $\{|x| \mid x \in \mathbb{K}\}$.

During the forties, Mark Krasner had the idea of introducing a kind of analytic functions based upon the following property in complex analysis. Let $D$ be an open bounded subset of $\mathbb{C}$. Then, by Runge's Theorem, every holomorphic functions in $D$ which is continuous on the closure of $D$ is the uniform limit on $\bar{D}$ of sequences of rational functions with no pole in $\bar{D}$. This property suggested Marc Krasner defining analytic elements in a certain kind of sets called quasi-connected sets, as the uniform limit of a sequence of rational functions with no pole in such a set. Later, it appeared that such analytic elements may also be defined in a more general class of sets, particularly infraconnected sets. Recall that a subset of $\mathbb{K}$ is said to be infraconnected if for every $a \in D$, the closure of the image of the function $I_{a}$ defined in $D$ as $I_{a}(x)=|x-a|$, is an interval.

Given a closed bounded subset $D$ of $\mathbb{K}$, we denote by $\widetilde{D}$ the smallest closed disk containing $D$ i.e. if $R=\operatorname{diam}(\mathrm{D})$, then $\widetilde{D}=d(a, R)$ with $a \in D$.

${ }^{0} 2010$ Mathematics Subject Classification: 12J25; $46 \mathrm{~S} 10$

The second named author is supported by Vietnam's National Foundation for Science and Technology Development (NAFOSTED) under the Grant No. 101.04-2014.41. 
Lemma I.1.1 ([12], Lemma 1.10): let $D$ be a closed bounded subset of $\mathbb{I K}$. Then $\widetilde{D} \backslash D$ admits a partition of the form $\left\{d\left(a_{i}, r_{i}^{-}\right)_{i \in I}\right\}$ where each disk $d\left(a_{i}, r_{i}^{-}\right)$ is maximal.

Definitions and notation: Let $D$ be a closed bounded subset of $\mathbb{K K}$. The disks $d\left(a_{i}, r_{i}^{-}\right)$lying in the partition of $\widetilde{D} \backslash D$ shown above are called the holes of $D$.

Let $R(D)$ be the $\mathbb{K}$-algebra of rational functions without poles in $D$ provided with the norm of uniform convergence on $D$ and let $H(D)$ be the completion of $R(D)$ with respect to that norm. So, $H(D)$ is a $\mathbb{K}$-Banach algebra.

Now consider a closed unbounded subset $D$ of $\mathbb{K}$ and let $R_{b}(D)$ be the algebra of bounded rational functions having no pole in $D$, provided with the norm of uniform convergence on $D$. Then the completion of $R_{b}(D)$ is a $\mathbb{K}$-Banach algebra $H_{b}(D)$ again whose elements are called bounded analytic elements in D. Particularly this applies to sets of the form $\mathbb{K} \backslash d\left(a, r^{-}\right)$. Moreover we denote by $H_{0}(D)$ the IK-Banach algebra of elements $f$ such that $\lim _{|x| \rightarrow+\infty, x \in D} f(x)=0$.

Let us recall that a filter $\mathcal{F}$ on a set $E$ is said to be secant with a subset $B$ of $E$ if the family of sets $H \cap B, H \in \mathcal{F}$ makes a filter on $B$.

Here we will not recall the theory of analytic functions due to Krasner, soon generalized by Philippe Robba and next by Kamal Boussaf. But we have to describe some properties of analytic elements on infraconnected sets. Properties of these functions are nice because they derive from those of rational functions.

Particularly, the absolute value of an analytic element admits a limit along a circular filter and so, multiplicative semi-norms on the $\mathbb{K}$-algebra of the analytic elements on a bounded closed subset $D$ of $\mathbb{K}$ are characterized by circular filters on $D$. These properties explain the behavior of analytic and meromorphic functions in the whole field $\mathbb{K}$ and inside a disk [9], [10], [12].

Notation: Throughout this chapter, $D$ is a closed bounded infraconnected subset of $\mathbb{K}$. We will denote by $\operatorname{Mult}\left(H(D),\|.\|_{D}\right)$ the set of continuous multiplicative semi-norms $\psi$ of the $\mathbb{K}$-algebra $H(D)$.

Let $a \in \mathbb{K}$ and let $r^{\prime}, r^{\prime \prime}$ be such that $0<r^{\prime}<r^{\prime \prime}$. We denote by $\Gamma\left(a, r^{\prime}, r^{\prime \prime}\right)$ the annulus $\left\{x \in K\left|r^{\prime}<\right| x-a \mid<r^{\prime \prime}\right\}$ and by $\Delta\left(a, r^{\prime}, r^{\prime \prime}\right)$ the annulus $\left\{x \in K\left|r^{\prime} \leq\right| x-a \mid \leq r^{\prime \prime}\right\}$. Now, let $a \in \mathbb{K}, r>0$ and consider the filter admitting for basis the annuli $\Gamma\left(b, r^{\prime}, r^{\prime \prime}\right)$ with $b \in d(a, r)$ and $r^{\prime}<r<r^{\prime \prime}$. This filter is called circular filter of center $a$, of diameter $r$. Moreover if $\mathbb{K}$ is not spherically complete, each decreasing sequence of disks $\left(D_{n}\right)$ whose intersection is empty also defines a filter that is called circular filter of basis $\left(D_{n}\right)$. Finally, for every $a \in \mathbb{K}$, the filter of neighborhoods of $a$ is called circular filter of neighborhoods of a and such a circular filter is said to be punctual.

Theorem I.1.2 (B. Guennebaud, G. Garandel [E0], Theorem 13.1): Each circular filter $\mathcal{F}$ on $\mathbb{K}$ defines a multiplicative semi-norm on $\mathbb{K}[x]$ which is a norm if and only if it is not punctual and the semi-norm is continuous with respect to the norm $\|.\|_{D}$ if and only if the filter is secant with $D$. Each circular filter $\mathcal{F}$ secant with $D$ defines on $\left(R(D),\|.\|_{D}\right)$ a continuous multiplicative semi-norm $\varphi_{\mathcal{F}}$ that has continuation to $H(D)$ and the mapping associating to each circular filter secant with $D$, its multiplicative semi-norm $\varphi_{\mathcal{F}}$ is a bijection from the set of circular filters 
secant with $D$ onto the set of continuous multiplicative semi-norms on $H(D)$ and on $R(D)$. Mult $\left(H(D),\|.\|_{D}\right)$ is compact with respect to the topology of pointwise convergence.

The following theorem is easily proven and is given as Corollary 14.7 in [10]:

Theorem I.1.3: $A$ set $D$ is infraconnected if and only if $\operatorname{Mult}\left(R(D),\|.\|_{D}\right)$ is connected.

\section{I.2. Power and Laurent series}

All analytic elements in $d\left(0, R^{-}\right)$are power series converging in $d\left(0, R^{-}\right)$. The converse is false. However, concerning a "closed " disk $d(0, R)$, we will see that the analytic elements in $d(0, R)$ are exactly the power series converging in this disk.

Definitions: Let $f(x)=\sum_{n=0}^{\infty} a_{n} x^{n}$ be a power series with coefficients in $\mathbb{K}$.

As usual, when $\limsup _{n \rightarrow \infty} \sqrt[n]{\left|a_{n}\right|} \neq 0$, we call radius of convergence of $f$ the number $r=\frac{1}{\limsup _{n \rightarrow \infty} \sqrt[n]{\left|a_{n}\right|}} \quad$ (with $r=0$ when $\limsup _{n \rightarrow \infty} \sqrt[n]{\left|a_{n}\right|}=+\infty$ ).

When $\limsup _{n \rightarrow \infty} \sqrt[n \rightarrow \infty]{\left|a_{n}\right|}=0$, we define the radius of convergence of $f$ as $+\infty$

Example: Let $f(x)=\sum_{n=1}^{\infty} n x^{n}$. The radius of convergence of this series is 1 . This function obviously defines the rational function $\frac{x}{(1-x)^{2}}$ in $d\left(1,1^{-}\right)$.

Notations and definitions: Power series whose radius of convergence is $\infty$ are called entire functions on $\mathbb{K}$ and the set of entire functions is denoted by $\mathcal{A}(\mathbb{K})$.

For every $a \in \mathbb{K}, r \in \mathbb{R}_{+}^{*}$, similarly we denote by $\mathcal{A}\left(d\left(a, r^{-}\right)\right)$the set of power series in $x-a$ whose radius of convergence is superior or equal to $r$ and by $\mathcal{A}_{b}\left(d\left(a, r^{-}\right)\right)$the set of functions $f \in \mathcal{A}\left(d\left(a, r^{-}\right)\right)$that are bounded in $d\left(a, r^{-}\right)$. The set $\mathcal{A}\left(d\left(a, r^{-}\right)\right) \backslash \mathcal{A}_{b}\left(d\left(a, r^{-}\right)\right)$will be denoted by $\mathcal{A}_{u}\left(d\left(a, r^{-}\right)\right)$.

Similarly, we denote by $\mathcal{A}\left(\mathbb{K} \backslash d\left(a, r^{-}\right)\right)$the set of Laurent series converging whenever $|x-a| \geq r$ and by $\mathcal{A}_{b}\left(\mathbb{K} \backslash d\left(a, r^{-}\right)\right)$the $\mathbb{K}$-Banach algebra of bounded Laurent series converging whenever $|x-a| \geq r$.

Let $R \in \mathbb{R}_{+}^{*}$ and let $f \in \mathcal{A}\left(d\left(a, R^{-}\right)\right)$. Given $\left.r \in\right] 0, R[, f$ belongs to $H(d(a, r))$, hence for every circular filter $\mathcal{F}$ secant with $d(a, r), \varphi_{\mathcal{F}}(f)$ is defined. Given $b \in d(a, R)$, if $s<R$, and if $\mathcal{F}$ is the circular filter of center $b$ and diameter $s$, we put $\varphi_{b, s}(f)=\varphi_{\mathcal{F}}(f)=\|f\|_{d(b, s)}$. Particularly, if $a=0$ we put $|f|(s)=$ $\lim _{\mathcal{F}}|f(x)|=\lim _{|x| \rightarrow s,|x| \neq r}|f(x)|$.

The following theorem is important in the Nevanlinna Theory:

Theorem I.2.1 ([12], Theorem 14.7): Let $f \in \mathcal{A}(K)$. The following three statements are equivalent: 
i) $\lim _{r \rightarrow+\infty} \frac{|f|(r)}{r^{q}}=+\infty \forall q \in \mathbb{N}$,

ii) there exists no $q \in \mathbb{N}$ such that $\lim _{r \rightarrow+\infty} \frac{|f|(r)}{r^{q}}=0$,

iii) $f$ is not a polynomial.

Theorem I.2.2 ([12], Theorem 14.6) : Let $r \in \mathbb{R}_{+}^{*}$ and let $D=d(0, r)$. Then $H(D)$ is the set of power series $f(x)=\sum_{n=0}^{\infty} a_{n} x^{n}$ such that $\lim _{|n|_{\infty} \rightarrow \infty}\left|a_{n}\right| r^{n}=0$ and we have

$$
\|f\|_{D}=\max _{n \in \mathbb{N}}\left|a_{n}\right| r^{n}=\varphi_{\mathcal{F}}(f) .
$$

Moreover, the norms $\|\cdot\|_{d(0, r)}$ and $\|\cdot\|_{C(0, r)}$ are multiplicative and coincide on $H(C(0, r))$.

Theorem I.2.3 (Corollary 14.15): Let $f \in \mathcal{A}\left(d\left(0, r^{-}\right)\right)$be not identically zero. For every $\alpha \in d\left(0, r^{-}\right), f(x)$ is equal to a power series

$$
\sum_{n=0}^{\infty} b_{n}(\alpha)(x-\alpha)^{n}
$$

If $f$ is not identically zero and if $\alpha$ is a zero of $f$ in $d\left(0, r^{-}\right), \alpha$ is an isolated zero and $f$ factorizes in $\mathcal{A}\left(d\left(0, r^{-}\right)\right)$in the form $(x-\alpha)^{q} g(x)$, with $g \in \mathcal{A}\left(d\left(0, r^{-}\right)\right), q \in$ $\mathbb{N}^{*} g(\alpha) \neq 0$.

Definition: Let $D$ be an infraconnected subset of $\mathbb{K}$, let $f \in H(D)$, let $\alpha \in \stackrel{\circ}{D}$, let $r>0$ be such that $d(\alpha, r) \subset D$ and suppose $f(x)=\sum_{n=q}^{\infty} b_{n}(x-\alpha)^{n}$ whenever $x \in d(\alpha, r)$, with $b_{q}(\alpha) \neq 0$ and $q>0$. Then $\alpha$ is called a zero of multiplicity order $q$, or more simply, a zero of order $q$. In the same way, $q$ is called the multiplicity order of $\alpha$.

Theorem I.2.4 ([12], Theorem 14.19): Let $R \in \mathbb{R}_{+}^{*}$ and let $f \in \mathcal{A}\left(d\left(a, R^{-}\right)\right)$. Then $f$ is invertible in $\mathcal{A}\left(d\left(a, R^{-}\right)\right)$if and only if $f$ has no zero in $d\left(a, R^{-}\right)$.

Theorem I.2.5 ([12], Theorem 14.20): Let $R \in \mathbb{R}_{+}^{*}$. The $\mathbb{K}$-subalgebra $\mathcal{A}_{b}\left(d\left(0, R^{-}\right)\right)$of $\mathcal{A}\left(d\left(0, R^{-}\right)\right)$is a Banach $\mathbb{K}$-algebra with respect to the norm $\|\cdot\|_{d\left(0, R^{-}\right)}$. Further, this norm is multiplicative and satisfies $\|f\|_{d\left(0, R^{-}\right)}=\lim _{r \rightarrow R}|f|(r)=\sup _{n \in \mathbb{N}}\left|a_{n}\right| R^{n}$.

$$
\text { Let } f(x)=\sum_{n=0}^{\infty} a_{n} x^{n} \in \mathcal{A}\left(d\left(0, R^{-}\right)\right) \text {. Then } f \text { is bounded in } d\left(0, R^{-}\right) \text {if and only }
$$
if so is the sequence $\left(\left|a_{n}\right| R^{n}\right)_{n \in \mathbb{N}}$. Moreover, if $f$ is bounded, then $\|f\|_{d\left(0, R^{-}\right)}=$ $\sup _{n \in \mathbb{N}}\left|a_{n}\right| R^{n}$.

Corollary I.2.5.a: Let $R \in \mathbb{R}_{+}^{*}$ and let $f, g \in \mathcal{A}\left(d\left(a, R^{-}\right)\right)$. Then $f g$ belongs to $\mathcal{A}_{b}\left(d\left(a, R^{-}\right)\right)$if and only if so do both $f$ and $g$ and $\mathcal{A}_{b}\left(d\left(a, R^{-}\right)\right)$is $\mathbb{I K}$-subalgebra of $\mathcal{A}\left(d\left(a, R^{-}\right)\right)$. 
Theorem I.2.6 ([12], Theorem 14.22): Suppose that $\mathbb{K}$ has characteristic different from 2. Let $f, g \in \mathcal{A}(\mathbb{K}) \backslash \mathbb{K}$ (resp. $f, g \in \mathcal{A}_{u}\left(d\left(0, r^{-}\right)\right)$) be distinct. Then $f^{2}-g^{2}$ belongs to $\mathcal{A}(\mathbb{K}) \backslash \mathbb{K}$ (resp. $f, g \in \mathcal{A}_{u}\left(d\left(0, r^{-}\right)\right)$).

Theorem I.2.7 ([12], Theorem 14.23): For every $r \in \mathbb{R}_{+}^{*}, H\left(d\left(0, r^{-}\right)\right)$is included in $\mathcal{A}_{b}\left(d\left(0, r^{-}\right)\right)$.

Remarks: Actually, $H\left(d\left(0, r^{-}\right)\right)$is much smaller than $\mathcal{A}_{b}\left(d\left(0, r^{-}\right)\right)$. In particular, $\sqrt[q]{1+x}$ belongs to $\mathcal{A}_{b}\left(d\left(0,1^{-}\right)\right.$, but does not belong to $H\left(d\left(0,1^{-}\right)\right)$.

Let $\sum_{0}^{+\infty} a_{n} x^{n}$ be a power series whose radius of convergence is $r$. Suppose first that $r \in|\mathbb{K}|$. If there is at least one point $\alpha \in C(0, r)$ such that the series converges at $\alpha$, then this implies that $\lim _{n \rightarrow+\infty}\left|a_{n}\right| r^{n}=0$ and hence the series converges in all $C(0, r)$ and defines an element of $H(d(0, r))$. If $r$ does not belong to $|\mathbb{K}|$, the power series converging in $d(0, r)$ are just the power series converging in $d\left(0, r^{-}\right)$and hence, this is why we don't have to consider analytic functions inside a disk $d(a, r)$, whenever $r>0$.

\section{I.3. The Mittag-Leffler Theorem}

Throughout the paragraph I.3, $D$ is a closed infraconnected subset of $\mathbb{K}$. The wonderful Mittag-Leffler Theorem for analytic elements is due to Marc Krasner who showed it on quasi-connected sets. The same proof holds on infraconnected sets as it was shown by Philippe Robba. The theorem shows that a Banach space $H(D)$ is a direct topological sum of elementary subspaces and is indispensable to have a clear image of the space $H(D)$.

Theorem I.3.1: (M.Krasner, [19], [12], Theorem 15.1) Let $f \in H(D)$. There exists a unique sequence of holes $\left(T_{n}\right)_{n \in \mathbb{N}^{*}}$ of $D$ and a unique sequence $\left(f_{n}\right)_{n \in \mathbb{N}}$ in $H\left(\mathbb{K} \backslash T_{n}\right)$ such that $f_{0} \in H(\widetilde{D})(n>0), \quad \lim _{n \rightarrow \infty} f_{n}=0$ satisfying further

(1) $\quad f=\sum_{n=0}^{\infty} f_{n}$ and $\|f\|_{D}=\sup _{n \in \mathbb{N}}\left\|f_{n}\right\|_{D}$.

For every hole $T_{n}=d\left(a_{n}, r_{n}^{-}\right)$, we have

(2) $\quad\left\|f_{n}\right\|_{D}=\left\|f_{n}\right\|_{\mathbb{K} \backslash T_{n}}=\varphi_{a_{n}, r_{n}}\left(f_{n}\right) \leq \varphi_{a_{n}, r_{n}}(f) \leq\|f\|_{D}$.

If $D$ is bounded and if $\widetilde{D}=d(a, r)$ we have

(3) $\left\|f_{0}\right\|_{D}=\left\|f_{0}\right\|_{\widetilde{D}}=\varphi_{a, r}\left(f_{0}\right) \leq \varphi_{a, r}(f) \leq\|f\|_{D}$.

Let $D^{\prime}=\widetilde{D} \backslash\left(\bigcup_{n=1}^{\infty} T_{n}\right)$. Then $f$ belongs to $H\left(D^{\prime}\right)$ and its decomposition in $H\left(D^{\prime}\right)$ is given again by $(1)$ and $f$ satisfies $\|f\|_{D^{\prime}}=\|f\|_{D}$.

The Mittag-Leffler Theorem suggests some new definitions 
Definitions and notations: Let $f \in H(D)$. We consider the series $\sum_{n=0}^{\infty} f_{n}$ obtained in the last Theorem, whose sum is equal to $f$ in $H(D)$, with $f_{0} \in H(\widetilde{D}), f_{n} \in$ $H\left(\mathbb{K} \backslash T_{n}\right) \backslash\{0\}$ and with the $T_{n}$ holes of $D$. Each $T_{n}$ will be called a $f$-hole and $f_{n}$ will be called the Mittag-Leffler term of $f$ associated to $T_{n}$, whereas $f_{0}$ will be called the principal term of $f$. For each $f$-hole $T$ of $D$, the Mittag-Leffler term of $f$ associated to $T$ will be denoted by $\overline{\overline{f_{T}}}$ whereas the principal term of $f$ will be denoted by $\overline{\overline{f_{0}}}$. The series $\sum_{n=0}^{\infty} f_{n}$ will be called the Mittag-Leffler series of $f$ on the infraconnected set $D$.

Example 1: Let $f \in H\left(d\left(0,1^{-}\right)\right)$and let $\left(d\left(\alpha_{m}, 1^{-}\right)\right)_{m \in \mathbb{N}^{*}}$ be the family of $f$ holes. Then $f$ is of the form

$$
\text { (1) } \sum_{n=0}^{\infty} a_{n, 0} x^{n}+\sum_{m, n \in \mathbb{N}^{*}} \frac{a_{n, m}}{\left(x-\alpha_{m}\right)^{n}}
$$

with $\lim _{n \rightarrow \infty} a_{n, 0}=0, \lim _{n \rightarrow \infty}\left|a_{n, m}\right|=0$ whenever $m \in \mathbb{N}^{*}$ and $\lim _{m \rightarrow \infty}\left(\sup _{n \in \mathbb{N}^{*}}\left|a_{n, m}\right|\right)=0$. On the other hand, $f$ satisfies

$$
\|f\|_{d\left(0,1^{-}\right)}=\sup _{m \in \mathbb{N}, n \in \mathbb{N}^{*}}\left|a_{n, m}\right| .
$$

Conversely, every function of the form (1), with the $\alpha_{m}$ satisfying $\left|\alpha_{m}\right|=$ $\left|\alpha_{j}-\alpha_{m}\right|=1$ whenever $m \neq j$, belongs to $H\left(d\left(0,1^{-}\right)\right)$. The norm $\|\cdot\|_{d\left(0,1^{-}\right)}$is multiplicative and equal to $\varphi_{0,1}$.

Example 2: $\quad$ Let $r_{1}, r_{2} \in \mathbb{R}_{+}$satisfy $0<r_{1}<r_{2}$. Then $H\left(\Delta\left(0, r_{1}, r_{2}\right)\right)$ is equal to the set of the Laurent series $\sum_{-\infty}^{+\infty} a_{n} x^{n}$ with $\lim _{n \rightarrow-\infty}\left|a_{n}\right| r_{1}^{n}=\lim _{n \rightarrow \infty}\left|a_{n}\right| r_{2}^{n}=0$ and we have

$$
\left\|\sum_{-\infty}^{+\infty} a_{n} x^{n}\right\|_{\Delta\left(0, r_{1}, r_{2}\right)}=\max \left(\sup _{n \geq 0}\left|a_{n}\right| r_{1}^{n}, \sup _{n<0}\left|a_{n}\right| r_{2}^{n}\right) .
$$

Example 3: Let $r \in \mathbb{R}_{+}^{*}$. Then $H(C(0, r))$ is equal to the set of the Laurent series $\sum_{-\infty}^{+\infty} a_{n} x^{n}$ with $\lim _{|n|_{\infty} \rightarrow \infty}\left|a_{n}\right| r^{n}=0$ and we have $\left\|\sum a_{n} x^{n}\right\|_{C(0, r)}=\sup _{n \in \mathbb{Z}}\left|a_{n}\right| r^{n}$. Next, the norm $\|\cdot\|_{C(0, r)}$ is multiplicative and equal to $\varphi_{0, r}$.

Theorem I.3.2 ([12], Theorem 15.12): Let $D_{1}, D_{2}$ be infraconnected subsets of $\mathbb{K}$ having finitely many holes, such that $D_{1} \cap D_{2} \neq \emptyset$ and let $f_{j} \in H\left(D_{j}\right), j=1,2$ be such that $f_{1}(x)=f_{2}(x) \forall x \in D_{1} \cap D_{2}$. Then the function $f$ defined in $D_{1} \cup D_{2}$ as $f(x)=f_{j}(x) \forall x \in D_{j}, j=1,2$, belongs to $H\left(D_{1} \cup D_{2}\right)$.

Notation: Let $E$ be a $\mathbb{K}$-Banach space. We will denote by $E^{\oplus}$ the $\mathbb{K}$-Banach space of continuous linear forms of $E$ provided with its usual norm. The dual of a Banach space $H(D)$ was thoroughly studied by Yvette Amice (Theorem 15.13 in $[12])$. 
Theorem I.3.3 (Y. Amice, [12], Theorem 15.13): Let $r \in \mathbb{R}_{+}$. Given $h(t)=\sum_{n=0}^{\infty} \frac{b_{n}}{t^{n}} \in \mathcal{A}_{b}(\mathbb{K} \backslash d(0, r))$ there exists a unique $\phi_{h} \in H(d(0, r))^{\oplus}$ satisfying $\phi_{h}\left(x^{q}\right)=b_{q},(q \in \mathbb{N})$. Moreover, on the space $\mathcal{A}_{b}(\mathbb{K} \backslash d(0, r))$ provided with the norm $\|.\|_{\mathbb{K} \backslash d(0, r)}$, the mapping $h \rightarrow \phi_{h}$ is an isometric isomorphism from $\mathcal{A}_{b}(\mathbb{K} \backslash d(0, r))$ onto $H(d(0, r))^{\oplus}$.

Theorem I.3.4 ([12], Theorem 15.17): Let $f \in H_{b}\left(\mathbb{K} \backslash d\left(a, r^{-}\right)\right)$, and for each $\alpha \in d\left(a, r^{-}\right)$, let $f(x)=\sum_{n=0}^{\infty} \frac{b_{n}(\alpha)}{(x-\alpha)^{n}}$. Then $b_{1}(\alpha)$ does not depend on $\alpha$ in $d\left(a, r^{-}\right)$.

Definition and notation: Let $f \in H_{b}(D)$, let $T$ be a hole of $D$ and let $a \in T$. Let $f_{T}(x)=\sum_{n=1}^{\infty} \frac{b_{n}(a)}{(x-a)^{n}}$. Since $b_{1}(a)$ actually does not depend on $a$ in $T$, we set $\operatorname{res}(f, T)=b_{1}(a)$ and this number $\operatorname{res}(f, T)$ will be called the residue of $f$ on the hole $T$.

\section{I.4. Divisors of analytic functions}

\section{and Lazard's problem}

In this paragraph we shall define divisors in $\mathbb{K}$ or in a disk $d\left(a, R^{-}\right)$. We then shall define the divisor of an analytic function and of an ideal. Given a divisor $T$ on $\mathbb{K}$, there is no problem to construct an entire function whose divisor is $T$. But given a divisor $T$ on a disk $d\left(a, r^{-}\right)$, it is not always possible to find an analytic function (in that disk) whose divisor is $T$. This is Lazard's problem that we will recall.

Definition: We call a divisor in $\mathbb{K}$ (resp. a divisor in a disk $d\left(a, R^{-}\right)$) a mapping $T$ from $\mathbb{K}$ (resp. from $d\left(a, R^{-}\right)$) to $\mathbb{N}$ whose support is countable and has a finite intersection with each disk $d(a, r), \forall r>0$ (resp. $\forall r \in] 0, R[$ ). Thus, a divisor on $\mathbb{K}$ (resp. of $d\left(a, R^{-}\right)$) is characterized by a sequence $\left(a_{n}, q_{n}\right)_{n \in \mathbb{N}}$ with $a_{n} \in$ $\mathbb{K}, \lim _{n \rightarrow \infty}\left|a_{n}\right|=\infty$, (resp. $\left.a_{n} \in d\left(a, R^{-}\right), \lim _{n \rightarrow \infty}\left|a_{n}-a\right|=R\right),\left|a_{n}\right| \leq\left|a_{n+1}\right|$ and $q_{n} \in \mathbb{N}^{*} \forall n \in \mathbb{N}$. So, we will frequently denote a divisor by the sequence $\left(a_{n}, q_{n}\right)_{n \in \mathbb{N}}$ which characterizes it.

The set of divisors on $\mathbb{K}$ (resp. on $d\left(a, R^{-}\right)$) is provided with a natural multiplicative law that makes it a semi-group. It is also provided with a natural order relation: given two divisors $T$ and $T^{\prime}$, we can set $T \leq T^{\prime}$ when $T(\alpha) \leq T^{\prime}(\alpha) \forall \alpha \in$ $d\left(a, R^{-}\right)$. Moreover, if $T, T^{\prime}$ are two divisors such that $T(\alpha) \geq T^{\prime}(\alpha) \forall \alpha \in d\left(0, R^{-}\right)$, we can define the divisor $\frac{T}{T^{\prime}}$.

Given $f \in \mathcal{A}(\mathbb{K})$ (resp. $f \in \mathcal{A}\left(d\left(a, R^{-}\right)\right)$), we can define the divisor of $f$, denoted by $\mathcal{D}(f)$ on $K$ (resp. on $d\left(a, R^{-}\right)$) as $\mathcal{D}(f)(\alpha)=0$ whenever $f(\alpha) \neq 0$ and $\mathcal{D}(f)(\alpha)=s$ when $f$ has a zero of order $s$ at $\alpha$. 
Similarly, given an ideal $I$ of $\mathcal{A}(\mathbb{K})$ (resp. of $\mathcal{A}\left(d\left(a, R^{-}\right)\right)$) we will denote by $\mathcal{D}(I)$ the lower bound of the the $\mathcal{D}(f) f \in I$ and $\mathcal{D}(I)$ will be called the divisor of I.

Finally, given a divisor $T=\left(a_{n}, q_{n}\right)_{n \in \mathbb{N}}$, we shall denote by $\bar{T}$ the divisor $\left(a_{n}, 1\right)_{n \in \mathbb{N}}$. Let $T=\left(a_{n}, q_{n}\right)_{n \in \mathbb{N}}$ be a divisor on $\mathbb{K}$ (resp. of $d\left(a, R^{-}\right)$). For every $r>0$ (resp. $r \in] 0, R\left[\right.$ ) we set $|T|(r)=\prod_{\left|a_{j}\right| \leq r}\left(\frac{r}{\left|a_{j}\right|}\right)^{q_{j}}$. The divisor $T$ on $d\left(a, R^{-}\right)$is said to be bounded if $\lim _{r \rightarrow R}|T|(r)<\infty$ and then we put $\|T\|=\lim _{r \rightarrow R}|T|(r)$.

Remark: Let $f \in \mathcal{A}\left(d\left(a, R^{-}\right)\right)$and let $\left(a_{n}, q_{n}\right)_{n \in \mathbb{N}}=\mathcal{D}(f)$. Then $\omega_{a_{n}}(f)=$ $q_{n} \forall n \in \mathbb{N}$ and $\omega_{\alpha}(f)=0 \forall \alpha \in d\left(a, R^{-}\right) \backslash\left\{a_{n} \mid n \in \mathbb{N}\right\}$.

Theorem I.4.1 ([12], Theorem 28.1) Let $a \in \mathbb{K}, R>0$. Let $f, g \in \mathcal{A}(\mathbb{K})$ (resp. $f, g \in \mathcal{A}\left(\left(a, R^{-}\right)\right)$) be such that $\mathcal{D}(f) \geq \mathcal{D}(g)$. Then there exists $h \in \mathcal{A}(\mathbb{K})$ (resp. $h \in \mathcal{A}\left(d\left(a, R^{-}\right)\right)$) such that $f=g h$.

Corollary I.4.1.a: Let $a \in \mathbb{K}, R>0$. Let $I$ be an ideal of $\mathcal{A}(\mathbb{K})$ (resp. an ideal of $\mathcal{A}\left(d\left(a, R^{-}\right)\right)$) and suppose that there exists $g \in I$ such that $\mathcal{D}(g)=\mathcal{D}(I)$. Then $I=g \mathcal{A}(\mathbb{K})$ (resp. $\left.I=g \mathcal{A}\left(d\left(a, R^{-}\right)\right)\right)$.

In the whole field $\mathbb{K}$, given a divisor $T$, it is always possible to find an entire function admitting $T$ for divisor.

Theorem I.4.2 ([12], Theorem 28.4): Let $T=\left(\alpha_{n}, q_{n}\right)_{n \in \mathbb{N}}$ be divisor of $\mathbb{K}$ The infinite product $\prod_{n=1}^{\infty}\left(1-\frac{x}{\alpha_{n}}\right)^{q_{n}}$ is uniformly convergent in all bounded subsets of $\mathbb{K}$ and defines an entire function $f \in \mathcal{A}(\mathbb{K})$ such that $\mathcal{D}(f)=T$. Moreover, given $g \in \mathcal{A}(\mathbb{K})$ such that $\mathcal{D}(g)=T$, then $g$ is of the form $\lambda f$.

Corollary I.4.2.a: For every divisor $T$ on $\mathbb{K}$, there exists $f \in \mathcal{A}(\mathbb{K})$ such that $\mathcal{D}(f)=T$. Moreover, if $f(0)=1, f$ satisfies $|f|(r)=|T|(r) \forall r>0$.

So, given a divisor $T$ on $\mathbb{K}$, we can find an entire function whose divisor is just $T$. It is natural to consider the same problem inside a disk $d\left(a, r^{-}\right)$. Indeed, in $\mathbb{C}$, it is known that the similar problem always admits a solution, in the whole field $\mathbb{C}$ as well as inside an open disk. Actually, in the general context of a complete ultrametric algebraically closed field $\mathbb{K}$, given a divisor $T$ in $d\left(a, r^{-}\right)$, the problem of finding an analytic function $f$ in $d\left(a, r^{-}\right)$, admitting $T$ for divisor, has no solution when $\mathbb{I K}$ is not spherically complete, in the general case, but it has a solution when $\mathbb{K}$ is spherically complete, has proven by M. Lazard [20]. On the other hand, in the general case, we can construct an analytic function $f$ whose divisor is a little bit bigger than the given divisor $T$ but narrows it.

Theorem I.4.3 ([12], Theorem 28.14): Let $T=\left(a_{n}, q_{n}\right)_{n \in \mathbb{N}}$ be a divisor on the disk $d\left(a, R^{-}\right)$with $a_{n} \neq 0 \forall n \in \mathbb{N}$ and let $\epsilon>0$. There exists $f \in \mathcal{A}\left(d\left(a, R^{-}\right)\right)$ such that $\mathcal{D}(f) \geq T, f(0)=1$ and $|f|(r) \leq|T|(r)(1+\epsilon) \forall r \in] 0, R[$.

Remark: Here we may notice that $H\left(d\left(0, R^{-}\right)\right)$is smaller than $\mathcal{A}_{b}\left(d\left(0, R^{-}\right)\right)$. Indeed, there exist functions $f \in \mathcal{A}_{b}\left(d\left(0, R^{-}\right)\right)$having infinitely many zeros in $d\left(0, R^{-}\right)$. But any element of $H\left(d\left(0, R^{-}\right)\right)$is quasi-invertible and hence has finitely many zeros. 
Theorem I.4.4 (M. Lazard, [20], [12], Theorem 29.1 ): Let $\mathbb{K}$ be not spherically complete and let $\left(D_{n}\right)_{n \in \mathbb{N}}$ be a decreasing sequence of disks $d\left(u_{n}, \rho_{n}\right)$ such that $\bigcap_{n=0}^{\infty} D_{n}=\emptyset$. Let $R=\frac{1}{\lim _{n \rightarrow \infty} \rho_{n}}$. There exists sequences $\left(c_{n}\right)_{n \in \mathbb{N}}$ of $d\left(0, R^{-}\right)$such that $\lim _{n \rightarrow \infty}\left|c_{n}\right|=R$ and such that no function $f \in \mathcal{A}\left(d\left(0, R^{-}\right)\right)$admits for divisor the divisor $T=\left(c_{n}, 1\right)_{n \in \mathbb{N}}$.

Theorem I.4.5 (M. Lazard [20], [12], Theorem 29.4): Suppose $\mathbb{K}$ is spherically complete. Assume that $\left|Q_{m}\right|\left(\rho_{m}\right) \leq|T|\left(\rho_{m}\right) \forall m \in \mathbb{N}$. Let $\left.R \in\right] 0,+\infty[$. There exists $f \in \mathcal{A}\left(d\left(0, R^{-}\right)\right)$satisfying

i) $f(0)=1$,

ii) $|f|(r) \leq|T|(r) \forall r<R$,

iii) $P_{m}$ divides $f-Q_{m}$ in $\mathcal{A}\left(d\left(0, R^{-}\right)\right)$.

Corollary I.4.5.a: Suppose $\mathbb{K}$ is spherically complete. Let $T$ be a divisor on $d\left(a, R^{-}\right)$. There exists $f \in \mathcal{A}\left(d\left(a, R^{-}\right)\right)$such that $\mathcal{D}(f)=T$.

\section{I.5. Motzkin factorization,}

The idea of factorizing quasi-invertible analytic elements into a product of singular factors is a remarkable idea due to E. Motzkin [22]. This factorization has tight links with the Mittag-Leffler series. In the paragraph, we denote by $D$ an infraconnected open closed bounded subset of $\mathbb{K}$.

According to the properties of power series [12], we have first the following lemma.

Lemma I.5.1: Let $E=\mathbb{K} \backslash d\left(a, r^{-}\right)$with $a \in \mathbb{K}$ and $r>0$. Let $f \in H(E)$ be invertible in $H(E)$. Then $f(x)$ is a Laurent series of the form $\sum_{-\infty}^{q} a_{n}(x-a)^{n}$ with $\left|a_{q}\right| r^{q}>\left|a_{n}\right| r^{n} \forall n<q$.

Theorem I.5.2 derives from Lemma I.5.1:

Theorem I.5.2 ([12], Lemma 31.1): $\quad$ Let $T=d\left(a, r^{-}\right)$, with $a \in \mathbb{K}$ and $r>0$, let $E=\mathbb{K} \backslash T$ and take $b \in T$. Let $g \in H(E)$ be invertible in $H(E)$. Then there exist $\lambda \in \mathbb{K}, q \in \mathbb{Z Z}$ and $h \in H(E)$ invertible in $H(E)$, satisfying $\|h-1\|_{E}<1$, $\lim _{x \mid \rightarrow+\infty} h(x)=1$ and $g(x)=\lambda(x-b)^{q} h(x)$. Moreover, $\lambda, q$ are respectively unique, satisfying those relations. Further, both $\lambda, q$ do not depend on $b$ in $T$.

Definitions: Let $E=\mathbb{K} \backslash d\left(a, r^{-}\right)$with $a \in \mathbb{K}$ and $r>0$. Let $f \in H(E)$ be invertible in $H(E)$ and let $\lambda(x-a)^{q} h(x)$ be the factorization given in the previous theorem. The integer $q$ will be named the index of $f$ associated to $d\left(a, r^{-}\right)$and will be denoted by $m\left(f, d\left(a, r^{-}\right)\right)$. If $\lambda=1$, the element $f$ will be called a pure factor associated to $d\left(a, r^{-}\right)$. Let $\mathcal{G}^{T}$ be the group of invertible elements of $H(\mathbb{K} \backslash T)$.

The following Corollary is then immediate: 
Corollary I.5.2.a: Let $T=d\left(a, r^{-}\right)$. The set of pure factors associated to $T$ is a sub-multiplicative group of the group $\mathcal{G}^{T}$. Further, every element of $\mathcal{G}^{T}$ is of the form $\lambda h$ with $h$ a pure factor associated to $T$ and $\lambda \in \mathbb{K}^{*}$.

Definition: Let $f$ belong to $H(D)$. Let $T$ be a hole of $D$ and let $h$ be a pure factor associated to $T$. If $\frac{f}{h}$ belongs to $H(D \cup T)$ and has no zero inside $T, h$ is called Motzkin factor of $f$ in the hole $T$ and the number $m(h, T)$ is called Motzkin index of $f$ associated to $T$.

Theorem I.5.3 ([12], Theorem 31.16): Let $f \in H(D)$. For each hole $T$ of $D, f$ admits a unique Motzkin factor $f^{T}$. Moreover, the set of holes $T$ such that $f^{T} \neq 1$ is a sequence $\left(f^{T_{n}}\right)_{n \in \mathbb{N}}$ converging to 1 in $H(D)$, the set of indices $n$ such that $m\left(f, T_{n}\right) \neq 0$ is finite and $f$ is equal to the infinite product $f^{0} \prod_{n \in I} f^{T_{n}}$, that converges in $H(D)$, with $f^{0}$ a power series converging in $\widetilde{D}$ and I a countable set.

\section{I.6. Meromorphic functions}

In this chapter, we will define and examine the basic properties of meromorphic functions: relations with poles of analytic elements, absolute values on fields of meromorphic functions defined by circular filters, values of the derivative on a circular filter, developement in a Laurent series in an annulus, existence of primitives.

Definitions and notation: We denote by $\mathcal{M}(\mathbb{K})$ the field of fractions of $\mathcal{A}(\mathbb{K})$. The elements of $\mathcal{M}(\mathbb{K})$ are called meromorphic functions in $\mathbb{K}$.

In the same way, given $a \in \mathbb{K}$ and $r>0$, we denote by $\mathcal{M}\left(d\left(a, r^{-}\right)\right)$(resp. $\mathcal{M}_{b}\left(d\left(a, r^{-}\right)\right)$, resp. $\left.\mathcal{M}_{u}\left(d\left(a, r^{-}\right)\right)\right)$the field of fractions of $\mathcal{A}\left(d\left(a, r^{-}\right)\right)$(resp. the field of fractions of $\mathcal{A}_{b}\left(d\left(a, r^{-}\right)\right)$, resp. the set $\left.\mathcal{M}\left(d\left(a, r^{-}\right)\right) \backslash \mathcal{M}_{b}\left(d\left(a, r^{-}\right)\right)\right)$. The elements of $\mathcal{M}\left(d\left(a, r^{-}\right)\right)$are called meromorphic functions in $d\left(a, r^{-}\right)$.

Let $b \in \mathbb{K}$ (resp. $b \in d\left(a, R^{-}\right)$) and let $r \in \mathbb{R}_{+}^{*}$ (resp. $\left.r \in\right] 0, R[$ ). The absolute value $\varphi_{b, r}$ defined on $\mathcal{A}(\mathbb{K})$ (resp. on $\mathcal{A}\left(d\left(a, R^{-}\right)\right)$) has an immediate continuation to $\mathcal{M}(\mathbb{K})$ (resp. to $\mathcal{M}\left(d\left(a, R^{-}\right)\right)$) that we shall denote again by $\varphi_{b, r}$. In the same way, $\varphi_{0, r}$ will be denoted by $|\cdot|(r)$ on $\mathcal{M}(\mathbb{K})$ and on $\mathcal{M}\left(d\left(0, R^{-}\right)\right)$.

Let $f=\frac{h}{l} \in \mathcal{M}(\mathbb{K})$ (resp. $f=\frac{h}{l} \in \mathcal{M}\left(d\left(a, R^{-}\right)\right)$). For each $\alpha \in \mathbb{K}$ (resp. $\left.\alpha \in d\left(a, R^{-}\right)\right)$the number $\omega_{\alpha}(h)-\omega_{\alpha}(l)$ does not depend on the functions $h, l$ choosed to make $f=\frac{h}{l}$. Thus, we can generalize the notation by setting $\omega_{\alpha}(f)=$ $\omega_{\alpha}(h)-\omega_{\alpha}(l)$.

If $\omega_{\alpha}(f)$ is an integer $q>0, \alpha$ is called a zero of $f$ of order $q$.

If $\omega_{\alpha}(f)$ is an integer $q<0, \alpha$ is called a pole of $f$ of order $-q$.

If $\omega_{\alpha}(f) \geq 0, f$ will be said to be holomorphic at $\alpha$.

Similarly as for $\mathcal{A}(\mathbb{K})$, given $f \in \mathcal{M}(\mathbb{K})$ (resp. $f \in \mathcal{M}\left(d\left(a, R^{-}\right)\right)$), we can define the divisor $\mathcal{D}(f)$ on $\mathbb{K}$ (resp. of $d\left(a, R^{-}\right)$) as $\mathcal{D}(f)(\alpha)=0$ whenever $f(\alpha) \neq 0$ and $\mathcal{D}(f)(\alpha)=s$ when $f$ has a zero of order $s$ at $\alpha$. 
Lemma I.6.1 ([12], Lemma 32.3): Let $f \in \mathcal{M}(\mathbb{K})$. There exists $h \in \mathcal{A}(\mathbb{K})$ such that $\mathcal{D}(h)=\mathcal{D}(f)$ and then the function $l=\frac{h}{f}$ belongs to $\mathcal{A}(\mathbb{K})$. Then $\mathcal{D}\left(\frac{1}{f}\right)=\mathcal{D}(l)$ and we can write $f$ in the form $\frac{h}{l}$ with $h, l \in \mathcal{A}(\mathbb{K})$, having no
common zero.

Theorem I.6.2 ([12], Theorem 32.4): $\quad$ Let $f \in \mathcal{M}(\mathbb{K})$ (resp. $f \in \mathcal{M}\left(d\left(a, R^{-}\right)\right)$) have no pole in $\mathbb{K}$ (resp. in $d\left(a, R^{-}\right)$). Then $f$ belongs to $\mathcal{A}(\mathbb{K})$ (resp. to $\left.\mathcal{A}\left(d\left(a, R^{-}\right)\right)\right)$.

Corollary I.6.2.a: Let $f, g \in \mathcal{A}(\mathbb{K})$ (resp. $f, g \in \mathcal{A}\left(d\left(a, R^{-}\right)\right.$)) be such that $\mathcal{D}(g) \leq \mathcal{D}(f)$. There exists $h \in \mathcal{A}(\mathbb{K})$ (resp. $h \in \mathcal{A}\left(d\left(a, R^{-}\right)\right)$) such that $f=g h$.

Corollary I.6.2.b: Let $f \in \mathcal{M}(\mathbb{K})$ (resp. $f \in \mathcal{M}\left(d\left(a, R^{-}\right)\right)$) have no zero and no pole in $\mathbb{K}$ (resp. in $\left.d\left(a, R^{-}\right)\right)$. Then it is a constant (resp. an invertible element of $\mathcal{A}_{b}\left(d\left(a, R^{-}\right)\right)$).

Theorem I.6.3 ([12], Corollary 32.10): Let $\mathbb{K}$ be spherically complete, let $a \in \mathbb{K}, r \in \mathbb{R}_{+}^{*}$ and let $f \in \mathcal{M}\left(d\left(a, R^{-}\right)\right)$. There exist $g, h \in \mathcal{A}\left(d\left(a, R^{-}\right)\right)$, having no common zero, such that $f=\frac{g}{h}$.

Remark: If $\mathbb{K}$ is not spherically complete, in the general case, we cannot find an analytic function $h \in \mathcal{A}\left(d\left(a, R^{-}\right)\right)$such that $\mathcal{D}(h)=\mathcal{D}(f)$. Consequently, in a field such as $\mathbb{C}_{p}$, we can't write $f$ in the form $f=\frac{h}{l}$ with $h, l \in \mathcal{A}\left(d\left(a, R^{-}\right)\right)$, having no common zero (this gap was forgotten in several works).

However, we can take an algebraically closed spherically complete extension $\widehat{\mathbb{K}}$ of $\mathbb{K}$ and consider $f$ as an analytic function on the disk $\widehat{d}\left(a, R^{-}\right)$in the field $\widehat{\mathbb{K}}$ : then $f$ may be written in the form $f=\frac{\widehat{h}}{\widehat{l}}$ with $\widehat{h}, \widehat{l} \in \mathcal{A}\left(\widehat{d}\left(a, R^{-}\right)\right)$, with $\widehat{h}, \widehat{l}$ having
no common zero.

Definitions: Let $f \in \mathcal{M}(\mathbb{K})$ (resp. $f \in \mathcal{M}_{u}\left(d\left(a, R^{-}\right)\right)$) and let $b \in \mathbb{K}$. Then $b$ will be said to be an exceptional value for $f$ if $f-b$ has no zero in $\mathbb{K}$ (resp. in $\left.d\left(a, R^{-}\right)\right)$and $b$ will be said to be a pseudo-exceptional value for $f$ if $\lim _{r \rightarrow \infty}|f-b|(r)=0$ (resp. $\lim _{r \rightarrow R^{-}}|f-b|(r)=0$ ).

Moreover, if $f \in \mathcal{M}(\mathbb{K}) \backslash \mathbb{K}(x)$ (resp. if $f \in \mathcal{M}_{u}\left(d\left(a, R^{-}\right)\right)$), $b$ will be said to be a quasi-exceptional value for $f$ if $f-b$ has finitely many zeros in $\mathbb{K}$ (resp. in $\left.d\left(a, R^{-}\right)\right)$. 
Theorem I.6.4 ([12], Theorem 32.21): Let $f \in \mathcal{M}(\mathbb{K}) \backslash \mathbb{K}$, (resp. $f \in$ $\left.\mathcal{M}_{u}\left(d\left(a, R^{-}\right)\right)\right)$. If $b$ is an exceptional value for $f$ then it is a pseudo-exceptional value for $f$. Let $f \in \mathcal{M}(\mathbb{K}) \backslash \mathbb{K}(x)$, (resp. $f \in \mathcal{M}_{u}\left(d\left(a, R^{-}\right)\right.$)). If $b$ is a quasiexceptional value for $f$ then it is a pseudo-exceptional value for $f$.

Theorem I.6.5 ([12], Theorem 32.22): Let $f \in \mathcal{M}(\mathbb{K}) \backslash \mathbb{K}$, (resp. $f \in$ $\left.\mathcal{M}_{u}\left(d\left(a, R^{-}\right)\right)\right)$. Then $f$ admits at most one pseudo-exceptional value. Moreover, if $f \in \mathcal{A}(\mathbb{K}) \backslash \mathbb{K}$, (resp. $f \in \mathcal{A}\left(d\left(a, R^{-}\right)\right)$), then $f$ has no pseudo-exceptional value.

Corollary I.6.5.a: Let $f \in \mathcal{M}(\mathbb{K}) \backslash \mathbb{K}$, (resp. $f \in \mathcal{M}_{u}\left(d\left(a, R^{-}\right)\right)$). Then $f$ admits at most one exceptional value. Moreover, if $f \in \mathcal{M}(\mathbb{K}) \backslash \mathbb{K}(x)$, (resp. $\left.f \in \mathcal{M}_{u}\left(d\left(a, R^{-}\right)\right)\right)$, then $f$ admits at most one quasi-exceptional value. Further, if $f \in \mathcal{A}(\mathbb{K}) \backslash \mathbb{K}$ (resp. if $f \in \mathcal{A}_{u}\left(d\left(a, R^{-}\right)\right)$) then $f$ admits no exceptional value. And if $f \in \mathcal{A}(\mathbb{K}) \backslash \mathbb{K}[x]$ (resp. if $f \in \mathcal{A}_{u}\left(d\left(a, R^{-}\right)\right)$) then $f$ admits no quasi-exceptional value.

Definition and notation: Let $f \in \mathcal{M}(\mathbb{K})$ (resp. $f \in \mathcal{M}\left(d\left(0, R^{-}\right)\right)$have a pole $\alpha$ of order $q$ and let $f(x)=\sum_{k=-q}^{-1} a_{k}(x-\alpha)^{k}+h(x)$ with $a_{-q} \neq 0$ and $h \in \mathcal{M}(\mathbb{K})$ (resp. $f \in \mathcal{M}\left(d\left(0, R^{-}\right)\right)$and $h$ holomorphic at $\alpha$. Accordingly to usual notations the coefficient $a_{-1}$ is called residue of $f$ at $\alpha$ and denoted by $\operatorname{res}(f, \alpha)$.

We can now compare residues on a hole defined for analytic elements and residues at a point, we just defined for a meromorphic function:

Theorem I.6.6 ([12], Theorem 33.1): Let $a \in \mathbb{K}$, let $R \in \mathbb{R}_{+}^{*}$, let $f \in$ $\mathcal{M}\left(d\left(a, R^{-}\right)\right)$and let $\left.r \in\right] 0, R\left[\right.$. Let $\alpha_{j}, 1 \leq j \leq q$ be the poles of $f$ in $d(a, r)$, let $\rho \in] 0, \min _{i \neq j}\left|\alpha_{i}-\alpha_{j}\right|\left[\right.$ and for each $j=1, \ldots, q$, let $\left.\rho_{j} \in\right] 0, \rho\left[\right.$, let $T_{j}=d\left(\alpha_{j}, \rho_{j}^{-}\right)$. Let $D=d(\alpha, r) \backslash\left(\bigcup_{j=1}^{q} T_{j}\right)$. Then $f$ belongs to $H(D)$ and $\operatorname{res}\left(f, \alpha_{j}\right)=\operatorname{res}\left(f, T_{j}\right), j=$ $1, \ldots, q$.

Corollary I.6.6.a: $\quad$ Let $f \in H_{b}(D)$ be meromorphic in $T=d\left(b, r^{-}\right)$and admit only one pole $b$ inside $T$. Let $q$ be the multiplicity order of $b$. Then the MittagLeffler term of $f$ associated to $T$ is of the form $\sum_{j=1}^{q} \frac{a_{j}}{(x-b)^{j}}$, with $a_{q} \neq 0$ and also is of the form $\frac{P}{\left(x-a_{j}\right)^{q}}$ where $P$ is a polynomial of degree $s<q$. Moreover, it does not depend on $r$ when $r$ tends to 0 .

It seems obvious that the condition for a meromorphic function to admit primitives is that all residues are null. Actually, the proof is not this immediate.

Theorem I.6.7 ([12], Theorem 33.12): $\quad$ A function $f \in \mathcal{M}((\mathbb{K})$ (resp. $f \in$ $\mathcal{M}\left(\left(d\left(a, R^{-}\right)\right), a \in \mathbb{K}, R>0\right)$ admits primitives in $\mathcal{M}(\mathbb{K})$ (resp. in $\mathcal{M}\left(\left(d\left(a, R^{-}\right)\right)\right)$ if and only if all residues of $f$ are nul.

Corollary I.6.7.a: $\quad$ Let $f \in \mathcal{M}\left((\mathbb{K})\right.$ (resp. $f \in \mathcal{M}\left(\left(d\left(a, R^{-}\right)\right), \quad a \in \mathbb{K}, R>0\right)$. Then $f^{\prime}$ belongs to $\mathbb{K}(x)$ if and only if so does $f$. 
The following Theorem is a strong improvement of the classical upper bound of $\left|f^{\prime}\right|(r)$ in function of $|f|(r)$ that is due to J.P. Bézivin [2]. The theorem is particularly useful for examining the derivative admitting no zero in $\mathbb{I K}$.

Theorem I.6.8 ([12], Theorem 33.10): Let $f \in \mathcal{M}\left(d\left(0, R^{-}\right)\right)$. For each $n \in \mathbb{N}$ and for all $r \in] 0, R[$, we have

$$
\left|f^{(n)}\right|(r) \leq|n !| \frac{|f|(r)}{r^{n}}
$$

Here, we can recall this theorem obtained in whose proof is mainly due to Jean-Paul Bézivin [2]]:

Theorem I.6.9 ([12], Theorem 44.16): Let $f \in \mathcal{M}(\mathbb{K})$ and for each $r>0$, let $n(f, r)$ be the number of multiple poles of $f$ in $d(0, r)$. If there exists $c>0$ and $s \in \mathbb{N}$ such that $n(r, f) \leq c r^{s} \forall r>0$, then $f^{\prime}$ admits no quasi-exceptional value.

\section{I.7. Nevanlinna Theory in the classical context}

The Nevanlinna Theory was made by Rolf Nevanlinna on complex functions in the 1920th [23]. It consists of defining counting functions of zeros and poles of a meromorphic function $f$ and giving an upper bound for multiple zeros and poles of various functions $f-b, b \in \mathbb{C}$.

A similar theory for functions in a p-adic field was constructed by A. Boutabaa [4], after some previous works by Ha Huy Khoai [16]. The p-adic Nevanlinna Theory was first stated and correctly proved by Abedelbaki Boutabaa in $\mathcal{M}(\mathbb{K})$ in 1988. The theory was extended to functions in $\mathcal{M}\left(d\left(0, R^{-}\right)\right)$by taking into account Lazard's problem in 1999 [5].

Throughout the next paragraphs, we assume that, we denote by $I$ the interval $[t,+\infty[$ and by $J$ an interval of the form $[t, R[$ with $t>0$.

We have to introduce the counting function of zeros and poles of $f$, counting or not multiplicity. Here we will choose a presentation that avoids assuming that all functions we consider admit no zero and no pole at the origin.

Definitions: We denote by $Z(r, f)$ the counting function of zeros of $f$ in $d(0, r)$ in the following way.

Let $\left(a_{n}\right), 1 \leq n \leq \sigma(r)$ be the finite sequence of zeros of $f$ such that $0<\left|a_{n}\right| \leq$ $r$, of respective order $s_{n}$.

We set $Z(r, f)=\max \left(\omega_{0}(f), 0\right) \log r+\sum_{n=1}^{\sigma(r)} s_{n}\left(\log r-\log \left|a_{n}\right|\right)$ and so, $Z(r, f)$ is called the counting function of zeros of $f$ in $d(0, r)$, counting multiplicity.

In order to define the counting function of zeros of $f$ without multiplicity, we put $\overline{\omega_{0}}(f)=0$ if $\omega_{0}(f) \leq 0$ and $\overline{\omega_{0}}(f)=1$ if $\omega_{0}(f) \geq 1$.

Now, we denote by $\bar{Z}(r, f)$ the counting function of zeros of $f$ without multiplicity: 
$\bar{Z}(r, f)=\overline{\omega_{0}}(f) \log r+\sum_{n=1}^{\sigma(r)}\left(\log r-\log \left|a_{n}\right|\right)$ and so, $\bar{Z}(r, f)$ is called the counting function of zeros of $f$ in $d(0, r)$ ignoring multiplicity.

In the same way, considering the finite sequence $\left(b_{n}\right), 1 \leq n \leq \tau(r)$ of poles of $f$ such that $0<\left|b_{n}\right| \leq r$, with respective multiplicity order $t_{n}$, we put

$N(r, f)=\max \left(-\omega_{0}(f), 0\right) \log r+\sum_{n=1}^{\tau(r)} t_{n}\left(\log r-\log \left|b_{n}\right|\right)$ and then $N(r, f)$ is called the counting function of the poles of $f$, counting multiplicity.

Next, in order to define the counting function of poles of $f$ without multiplicity, we put $\overline{\overline{\omega_{0}}}(f)=0$ if $\omega_{0}(f) \geq 0$ and $\overline{\overline{\omega_{0}}}(f)=1$ if $\omega_{0}(f) \leq-1$ and we set

$\bar{N}(r, f)=\overline{\overline{\omega_{0}}}(f) \log r+\sum_{n=1}^{\tau(r)}\left(\log r-\log \left|b_{n}\right|\right)$ and then $\bar{N}(r, f)$ is called the counting function of the poles of $f$, ignoring multiplicity.

Now, we can define the Nevanlinna function $T(r, f)$ in $I$ or $J$ as $T(r, f)=$ $\max (Z(r, f), N(r, f))$ and the function $T(r, f)$ is called characteristic function of $f$ or Nevanlinna function of $f$.

Finally, if $S$ is a subset of $\mathbb{K}$ we will denote by $Z^{S}\left(r, f^{\prime}\right)$ the counting function of zeros of $f^{\prime}$, excluding those which are zeros of $f-a$ for any $a \in S$.

Remark: If we change the origin, the functions $Z, N, T$ are not changed, up to an additive constant.

Lemma I.7.1 ([12], Lemma 40.1): Let $\widehat{\mathbb{K}}$ be a complete algebraically closed extension of $\mathbb{K}$ whose absolute value extends that of $\mathbb{K}$ and let $f \in \mathcal{M}(\mathbb{K})$ (resp. let $\left.f \in \mathcal{M}\left(d\left(0, R^{-}\right)\right)\right)$. Let $\widehat{d}(0, R)=\{x \in \widehat{\mathbb{K}}|| x \mid<R\}$. The meromorphic function $\widehat{f}$ defined by $f$ in $\widehat{d}(0, R)$ has the same Nevanlinna functions as $f$.

Theorem I.7.2 ([12], Theorem 40.2): Let $f \in \mathcal{M}(\mathbb{K})\left(\right.$ resp. $\left.f \in \mathcal{M}\left(d\left(0, R^{-}\right)\right)\right)$ have no zero and no pole at 0 . Then

$$
\log (|f|(r))=\log (|f(0)|)+Z(r, f)-N(r, f) .
$$

Theorem I.7.3 ([12], Theorem 40.3): Let $f, g \in \mathcal{M}(\mathbb{K})$ (resp. $f, g \in$ $\left.\mathcal{M}\left(d\left(0, R^{-}\right)\right)\right)$. Then

$$
\begin{gathered}
Z(r, f g) \leq Z(r, f)+Z(r, g), N(r, f g) \leq N(r, f)+N(r, g), \\
T(r, f g) \leq T(r, f)+T(r, g), \\
T(r, f+g) \leq T(r, f)+T(r, g)+O(1) \\
\left.T(r, c f)=T(r, f) \forall c \in \mathbb{K}^{*}, T\left(r, \frac{1}{f}\right)=T(r, f)\right), \\
\left.T\left(r, \frac{f}{g}\right) \leq T(r, f)\right)+T(r, g) .
\end{gathered}
$$

Suppose now $f, g \in \mathcal{A}(\mathbb{K})$ (resp. $f, g \in \mathcal{A}\left(d\left(0, R^{-}\right)\right)$). Then $Z(r, f g)=$ $Z(r, f)+Z(r, g), T(r, f)=Z(r, f)) T(r, f g)=T(r, f)+T(r, g)+O(1)$ and $T(r, f+g) \leq \max (T(r, f), T(r, g))$. 
Moreover, if $\lim _{r \rightarrow+\infty} T(r, f)-T(r, g)=+\infty$ then $T(r, f+g)=T(r, f)$ when $r$ is big enough.

Lemma I.7.4 ([12], Lemm 40.4): Let $\alpha_{1}, \cdots, \alpha_{n} \in \mathbb{K}$ be pairwise distinct, let $P(u)=\prod_{i=1}^{n}\left(u-\alpha_{i}\right)$ and let $f \in \mathcal{M}\left(d\left(0, R^{-}\right)\right)$. Then $Z(r, P(f))=\sum_{i=1}^{n} Z\left(r, f-\alpha_{i}\right)$ and $\bar{Z}(r, P(f))=\sum_{i=1}^{n} \bar{Z}\left(r, f-\alpha_{i}\right)$.

Now we can derive the following lemma:

Lemma I.7.5 ([12], Lemm 40.5): Let $f \in \mathcal{M}(\mathbb{K})$. Then $f$ belongs to $\mathbb{K}(x)$ if and only if $T(r, f)=O(\log r)$.

Corollary I.7.5.a: Let $f \in \mathcal{M}(\mathbb{K})$ (resp. $f \in \mathcal{M}\left(d\left(0, R^{-}\right)\right)$). Then

$$
Z\left(r, \frac{f^{\prime}}{f}\right)-N\left(r, \frac{f^{\prime}}{f}\right) \leq-\log r+O(1) .
$$

Theorem I.7.6 ([12], Theorem 40.7): Let $f \in \mathcal{A}(\mathbb{K})$ (resp. $f \in \mathcal{A}\left(d\left(0, R^{-}\right)\right)$) and let $b \in \mathbb{K}$. Then $Z(r, f)=Z(r, f-b)+O(1) r \in I$ (resp. $r \in J)$.

Theorem I.7.7 (First Main Theorem) ([12], Theorem 40.8):

Let $f, g \in \mathcal{M}(\mathbb{K})$ (resp. let $f, g \in \mathcal{M}\left(d\left(0, R^{-}\right)\right)$). Then $T(r, f+b)=T(r, f)+O(1)$. Let $h$ be a Moebius function. Then $T(r, f)=T(r, h \circ f)+O(1)$. Let $P(X) \in \mathbb{K}[X]$. Then $T(r, P(f))=\operatorname{deg}(P) T(r, f)+O(1)$ and $T\left(r, f^{\prime} P(f) \geq T(r, P(f))\right.$.

Suppose now $f, g \in \mathcal{A}(\mathbb{K})$ (resp. $f, g \in \mathcal{A}\left(d\left(0, R^{-}\right)\right)$). Then $Z(r, f g)=$ $Z(r, f)+Z(r, g)$

$T(r, f)=Z(r, f)), T(r, f g)=T(r, f)+T(r, g)+O(1)$ and

$T(r, f+g) \leq \max (T(r, f), T(r, g))$.

Moreover, if $\lim _{r \rightarrow+\infty} T(r, f)-T(r, g)=+\infty$ then $T(r, f+g)=T(r, f)$ when $r$ is big enough.

Theorem I.7.8 ([12], Theorem 40.9): Let $f \in \mathcal{M}(\mathbb{K})$ (resp. $f \in \mathcal{M}\left(d\left(0, R^{-}\right)\right)$). There exists $\phi, \psi \in \mathcal{A}(\mathbb{K})$ (resp. $\phi, \psi \in \mathcal{A}\left(d\left(0, R^{-}\right)\right)$) such that $f=\frac{\phi}{\psi}$ and $\max (T(r, \phi), T(r, \psi)) \leq T(r, f)+O(1), r \in I($ resp. $(r \in J))$.

Theorem I.7.9 ([12], Theorem 40.10): $\quad$ Let $f \in \mathcal{M}\left(d\left(0, R^{-}\right)\right)$. Then $f$ belongs to $\mathcal{M}_{b}\left(d\left(0, R^{-}\right)\right)$if and only if $T(r, f)$ is bounded in $[0, R[$.

Corollary I.7.9.a: Let $f \in \mathcal{M}_{u}\left(d\left(a, R^{-}\right)\right)$, and let $h \in \mathcal{M}_{b}\left(d\left(a, R^{-}\right)\right), h \neq 0$. Then fh belongs to $\mathcal{M}_{u}\left(d\left(a, R^{-}\right)\right)$.

We can also derive this Corollary :

Corollary I.7.9.b: Let $f \in \mathcal{M}\left(d\left(a, R^{-}\right)\right)$and let $P \in \mathbb{K}[x]$. Then $P(f)$ belongs to $\mathcal{M}_{b}\left(d\left(a, R^{-}\right)\right)$if and only if so does $f$. sharing:

The following theorem has proven to be very useful in certain problems of value 
Theorem I.7.10 ([12], Theorem 40.14): Let $f \in \mathcal{M}(\mathbb{I K})$ (resp. $f \in \mathcal{M}\left(d\left(0, R^{-}\right)\right)$). Then $Z\left(r, f^{\prime}\right)-N\left(r, f^{\prime}\right) \leq Z(r, f)-N(r, f)-\log r+O(1), r \in I$, (resp. $\left.r \in J\right)$. Moreover, $N\left(r, f^{(k)}\right)=N(r, f)+k \bar{N}(r, f)+O(1), r \in I$ (resp. $\left.r \in J\right)$ and $Z\left(r, f^{(k)}\right) \leq Z(r, f)+k \bar{N}(r, f)-k \log r+O(1), r \in I \quad($ resp. $r \in J)$.

Corollary: I.7.10.a Let $f \in \mathcal{M}(\mathbb{K})$ (resp. $f \in \mathcal{M}\left(d\left(0, R^{-}\right)\right)$). Then $T\left(r, f^{(k)}\right) \leq(k+1) T(r, f)+O(1)(r \in I)$ (resp. $\left.r \in J\right)$.

Theorem I.7.11: Let $f \in \mathcal{M}(\mathbb{K})$ (resp. $f \in \mathcal{M}\left(d\left(0, R^{-}\right)\right)$). Then,

$$
T(r, f)-Z(r, f) \leq T\left(r, f^{\prime}\right)-Z\left(r, f^{\prime}\right)+O(1) .
$$

Lemma I.7.12 ([12], Lemma 40.17): Let $f \in \mathcal{M}(\mathbb{I K})$ (resp. $\left.f \in \mathcal{M}\left(d\left(0, R^{-}\right)\right)\right)$and let $G=\frac{f^{\prime}}{f}$. Then, $G$ satisfies $Z(r, G) \leq N(r, G)-\log r+O(1) r \in I \quad$ (resp. $(r \in J)$.

We can now prove the Second Main Theorem under different forms. The following Theorem is essential and directly leads to the theorems. That theorem was first given in [21] in a different form.

Theorem I.7.13 ([12], Theorem 40.19): $\quad$ Let $f \in \mathcal{M}(\mathbb{K})$ and let $a_{1}, \ldots, a_{q} \in \mathbb{K}$ be distinct. Then

$$
(q-1) T(r, f) \leq \max _{1 \leq k \leq q}\left(\sum_{j=1, j \neq k}^{q} Z\left(r, f-a_{j}\right)\right)+O(1) .
$$

Corollary I.7.13.a: $\quad$ Let $f \in \mathcal{M}(\mathbb{K})$ and let $a_{1}, \ldots, a_{q} \in \mathbb{K}$ be distinct. Then

$$
(q-1) T(r, f) \leq \sum_{j=1}^{q} Z\left(r, f-a_{j}\right)+O(1)
$$

Theorem I.7.14 ([12], Theorem 40.21): Let $f \in \mathcal{M}\left(d\left(0, R^{-}\right)\right)$and let $\tau_{1}, \ldots, \tau_{q} \in \mathcal{M}_{b}\left(d\left(0, R^{-}\right)\right)$be distinct. Then

$$
(q-1) T(r, f) \leq \max _{1 \leq k \leq q}\left(\sum_{j=1, j \neq k}^{q} Z\left(r, f-\tau_{j}\right)\right)+O(1) .
$$

Corollary I.7.14.a: Let $f \in \mathcal{M}\left(d\left(0, R^{-}\right)\right)$and let $\tau_{1}, \ldots, \tau_{q} \in \mathcal{M}_{b}\left(d\left(0, R^{-}\right)\right)$be distinct. Then

$$
(q-1) T(r, f) \leq \sum_{j=1}^{q} Z\left(r, f-\tau_{j}\right)+O(1) .
$$

Theorem I.7.15 ([12], Theorem 40.23): Let $\alpha_{1}, \ldots, \alpha_{q} \in \mathbb{K}$, with $q \geq 2$, let $S=\left\{\alpha_{1}, \ldots, \alpha_{q}\right\}$ and let $f \in \mathcal{M}(\mathbb{K})$ (resp. $f \in \mathcal{M}\left(d\left(0, R^{-}\right)\right)$). Then $(q-1) T(r, f) \leq \sum_{j=1}^{q} \bar{Z}\left(r, f-\alpha_{j}\right)+Z\left(r, f^{\prime}\right)-Z_{0}^{S}\left(r, f^{\prime}\right)+O(1) \forall r \in I \quad($ resp. $\forall r \in J)$.

Moreover, if $f$ belongs to $f \in \mathcal{A}(\mathbb{K})$ (resp. $\mathcal{A}\left(d\left(0, R^{-}\right)\right)$), then 
$q T(r, f) \leq \sum_{j=1}^{q} \bar{Z}\left(r, f-\alpha_{j}\right)+Z\left(r, f^{\prime}\right)-Z_{0}^{S}\left(r, f^{\prime}\right)+O(1) \quad \forall r \in I \quad($ resp. $\forall r \in J)$.

Remark: The last Theorem does not hold in complex analysis. Indeed, let $f$ be a meromorphic function in $\mathbb{C}$ omitting two values $a$ and $b$, such as $f(x)=\frac{e^{x}}{e^{x}-1}$. Then $Z(r, f-a)+Z(r, f-b)=0$.

Theorem I.7.16 (Second Main Theorem, [12] Theorem 40.24): Let $\alpha_{1}, \ldots, \alpha_{q} \in$ $\mathbb{K}$, with $q \geq 2$, let $S=\left\{\alpha_{1}, \ldots, \alpha_{q}\right\}$ and let $f \in \mathcal{M}(\mathbb{K})$ (resp. $f \in \mathcal{M}_{u}\left(d\left(0, R^{-}\right)\right)$). Then $(q-1) T(r, f) \leq \sum_{j=1}^{q} \bar{Z}\left(r, f-\alpha_{j}\right)+\bar{N}(r, f)-Z_{0}^{S}\left(r, f^{\prime}\right)-\log r+O(1) \quad \forall r \in I \quad$ (resp. $\forall r \in J)$.

Moreover, if $f$ belongs to $f \in \mathcal{A}(\mathbb{K})$ (resp. $\mathcal{A}\left(d\left(0, R^{-}\right)\right)$), then $(q-1) T(r, f) \leq \sum_{j=1}^{q} \bar{Z}\left(r, f-\alpha_{j}\right)-Z_{0}^{S}\left(r, f^{\prime}\right)+O(1) \quad \forall r \in I \quad($ resp. $\forall r \in J)$.

\section{I.8. Classical applications of the Nevanlinna Theory}

Theorem I.8.1 ([12], Theorem 41.1): Let $a_{1}, a_{2} \in \mathbb{K}$ and let $f, g \in \mathcal{A}(\mathbb{K})$ (with $a_{1} \neq a_{2}$ ) satisfy $f^{-1}\left(\left\{a_{i}\right\}\right)=g^{-1}\left(\left\{a_{i}\right\}\right)(i=1,2)$. Then $f=g$.

Theorem I.8.2 ([12], Theorem 41.2): $\quad$ Let $a_{1}, a_{2}, a_{3} \in \mathbb{K}$ (with $a_{i} \neq a_{j} \forall i \neq$ $j)$ and let $f, g \in \mathcal{A}_{u}\left(d\left(a, R^{-}\right)\right)$satisfy $f^{-1}\left(\left\{a_{i}\right\}\right)=g^{-1}\left(\left\{a_{i}\right\}\right)(i=1,2,3)$. Then $f=g$.

Theorem I.8.3 ([12], Theorem 41.3): Let $a_{1}, a_{2}, a_{3}, a_{4} \in \mathbb{K}$ (with $a_{i} \neq$ $\left.a_{j} \forall i \neq j\right)$ and let $f, g \in \mathcal{M}(\mathbb{K})$ satisfy $f^{-1}\left(\left\{a_{i}\right\}\right)=g^{-1}\left(\left\{a_{i}\right\}\right)(i=1,2,3,4)$. Then $f=g$.

Theorem I.8.4 ([12], Theorem 41.4): Let $a_{1}, a_{2}, a_{3}, a_{4}, a_{5} \in \mathbb{K}$ (with $a_{i} \neq$ $\left.a_{j} \forall i \neq j\right)$ and let $f, g \in \mathcal{M}_{u}\left(d\left(a, R^{-}\right)\right)$satisfy $f^{-1}\left(\left\{a_{i}\right\}\right)=g^{-1}\left(\left\{a_{i}\right\}\right) \quad(i=$ $1,2,3,4,5)$. Then $f=g$.

Defintion: A polynomial $P \in \mathbb{K}[x]$ is called a polynomial of uniqueness for a family of functions $\mathcal{F}$ if $P(f)=P(g)$ implies $f=g$ for all $f, g \in \mathcal{F}$.

Theorem I.8.5 ([12], Theorem 41.9): Let

$$
Q(x)=(n+2)(n+3) x^{n+3}-2(n+3)(n+1) x^{n+2}+(n+3)(n+2) x^{n+1} .
$$

Then $Q$ is a polynomial of uniqueness for $\mathcal{M}(\mathbb{K})$ whenever $n \geq 2$ and is a polynomial of uniqueness for $\mathcal{M}_{u}\left(d\left(a, R^{-}\right)\right)$whenever $n \geq 3, a \in \mathbb{K}, R>0$.

(This remarkable polynomial was presented by G. Frank and M. Reinders [14])

We can now apply the Second Main Theorem to obtain results concerning certain algebraic curves: 
Theorem I.8.6 ([12], Theorem 42.2): Let $\Lambda$ be a non-degenerate elliptic curve of equation $y^{2}=\left(x-a_{1}\right)\left(x-a_{2}\right)\left(x-a_{3}\right)$. There do not exist $g, f \in \mathcal{M}(\mathbb{K})$ such that $g(t)=y, f(t)=x, t \in \mathbb{K}$. Moreover, given $a \in \mathbb{K}, R>0$ there do not exist $g, f \in \mathcal{A}_{u}\left(d\left(a, R^{-}\right)\right)$such that $g(t)=y, f(t)=x, t \in \mathbb{K}$.

And similarly:

Theorem I.8.7: Let $a_{i} \in \mathbb{K}, i=1, \ldots, q, q \geq 4$ be pairwise distinct, let $c \in \mathbb{K}$ and let $R$ be $>0$. Let $m \in \mathbb{N}$ be $\geq 2$. There exist no $f, g \in \mathcal{M}(\mathbb{K}) \backslash \mathbb{K}$ such that $g(t)^{m}=\prod_{i=1}^{q}\left(f(t)-a_{i}\right)$.

Theorem I.8.8 ([12], Theorem 42.4): Let $a_{i} \in \mathbb{K}, i=1, \ldots, q, q \geq 5$ be pairwise distinct, let $c \in \mathbb{K}$ and let $R$ be $>0$. Let $m \in \mathbb{N}$ be $\geq 2$ and prime to $q$. There exist neither $f, g \in \mathcal{M}(\mathbb{K}) \backslash \mathbb{K}$ nor $f, g \in \mathcal{M}_{u}\left(d\left(c, R^{-}\right)\right.$) such that $g(t)^{m}=\prod_{i=1}^{q}\left(f(t)-a_{i}\right)$.

Example: Let $\Lambda$ be a curve of equation $y^{2}=P(x)$ with $\operatorname{deg}(P)=5, P$ admitting five distinct zeros. If two functions $f, g \in \mathcal{M}(K)$ satisfy $g(t)^{2}=P(f(t))$, then the two both functions $f, g$ are constant. And if $f, g \in \mathcal{M}\left(d\left(a, R^{-}\right)\right)$satisfy $g(t)^{2}=P(f(t))$, then the two both functions $f, g$ belong to $\mathcal{M}_{b}\left(d\left(a, R^{-}\right)\right)$.

Theorem I.8.9 ([12], Theorem 42.10): Let $f, g \in \mathcal{M}(\mathbb{K})$ satisfy $g^{m}+f^{n}=$ 1 , with $\min (m, n) \geq 2$ and $\max (m, n) \geq 3$. Then $f$ and $g$ are constant. Moreover, if $f, g \in \mathcal{A}(\mathbb{K})$ and satisfy $g^{m}+f^{n}=1$, with $\min (m, n) \geq 2$, then $f$ and $g$ are constant.

Let us now recall the famous Hayman conjecture concerning complex meromorphic functions. Let $f$ be a function meromorphic on $\mathbb{C}$. W. Hayman showed that for every $n \geq 3, f^{\prime} f^{n}$ takes every value infinitely many times and he conjectured that this was true for all $n \geq 1$. That was proven for $n=2$ by E. Mues and for $n=1$ by W. Bergweiler and A. Eremenko [1] and separately by H.Chen and M. Fang [7].

On the field $\mathbb{K}$, we can consider the same problem. Jacqueline Ojeda [24] (and separately y H. Jurvanen) solved the problem for $n \geq 3$ also. Moreover, applying the Nevanlinna theory inside a disk, Jacqueline Ojeda also proved the same for unbounded meromorphic functions inside a disk $d\left(a, R^{-}\right)$. In 2013, Jacqueline Ojeda and I proved the conjecture for meromorphic functions on $\mathbb{K}$ when $n=2$ [13]. The conjecture remains for $n=1$.

Theorem I.8.10 ([24], [13], [12], Corollary 45.10 and Theorem 45.15,) Let $a \in \mathbb{K}$ and $R>0$. Let $f \in \mathcal{M}(\mathbb{K}) \backslash \mathbb{K}(x)$ (resp. $f \in \mathcal{M}_{u}\left(a, R^{-}\right)$). For every $n \geq 3, f^{\prime} f^{n}$ takes every value infinitely many times. Given $f \in \mathcal{M}(\mathbb{K}) \backslash \mathbb{K}(x)$, $f^{\prime} f^{2}$ takes every value infinitely many times. 


\section{I.9. Small functions}

Small functions with respect to a meromorphic function are well known in the general theory of complex functions. Particularly, one knows the Nevanlinna Theorem on 3 small functions. Here we will construct a similar theory.

Definitions and notation: Throughout the chapter we set $a \in K$ and $R \in$ ] $0,+\infty\left[\right.$. For each $f \in \mathcal{M}(\mathbb{K})$ (resp. $f \in \mathcal{M}\left(d\left(a, R^{-}\right)\right)$) we denote by $\mathcal{M}_{f}(\mathbb{K})$, (resp. $\left.\mathcal{M}_{f}\left(d\left(a, R^{-}\right)\right)\right)$the set of functions $h \in \mathcal{M}(\mathbb{K})$, (resp. $h \in \mathcal{M}\left(d\left(a, R^{-}\right)\right)$) such that $T(r, h)=o(T(r, f))$ when $r$ tends to $+\infty$ (resp. when $r$ tends to $R$ ). Similarly, if $f \in \mathcal{A}(\mathbb{K})$ (resp. $f \in \mathcal{A}\left(d\left(a, R^{-}\right)\right)$) we shall denote by $\mathcal{A}_{f}(\mathbb{K})$ (resp. $\left.\mathcal{A}_{f}\left(d\left(a, R^{-}\right)\right)\right)$the set $\mathcal{M}_{f}(\mathbb{K}) \cap \mathcal{A}(\mathbb{K}),\left(\right.$ resp. $\left.\mathcal{M}_{f}\left(d\left(a, R^{-}\right)\right) \cap \mathcal{A}\left(d\left(a, R^{-}\right)\right)\right)$.

The elements of $\mathcal{M}_{f}(\mathbb{K})$ (resp. $\mathcal{M}_{f}\left(d\left(a, R^{-}\right)\right)$) are called small meromorphic functions with respect to $f$, small functions in brief. Similarly, if $f \in \mathcal{A}(\mathbb{K})$ (resp. $\left.f \in \mathcal{A}\left(d\left(a, R^{-}\right)\right)\right)$the elements of $\mathcal{A}_{f}(\mathbb{K})$ (resp. $\mathcal{A}_{f}\left(d\left(a, R^{-}\right)\right)$) are called small analytic functions with respect to $f$ small functions in brief.

Remark: In [25], Yamanoi proved a Nevanlinna theorem on $n$ small complex meromorphic functions. Unfortunately, no similar theorem exists for functions in $\mathbb{K}$.

Theorem I.9.1 ([12], Theorem 43.1): Let $a \in \mathbb{K}$ and $r>0$. $\mathcal{A}_{f}(\mathbb{K})$ is a $\mathbb{K}$-subalgebra of $\mathcal{A}(\mathbb{K}), \mathcal{A}_{f}\left(d\left(a, R^{-}\right)\right)$is a $\mathbb{K}$-subalgebra of $\mathcal{A}\left(d\left(a, R^{-}\right)\right), \mathcal{M}_{f}(\mathbb{K})$ is a subfield of $\mathcal{M}(\mathbb{K})$ and $\mathcal{M}_{f}\left(d\left(a, R^{-}\right)\right)$is a subfield of field of $\left.\mathcal{M}\left(a, R^{-}\right)\right)$. Moreover, $\mathcal{A}_{b}\left(d\left(a, R^{-}\right)\right.$is a sub-algebra of $\mathcal{A}_{f}\left(d\left(a, R^{-}\right)\right.$and $\mathcal{M}_{b}\left(d\left(a, R^{-}\right)\right.$is a subfield of $\mathcal{M}_{f}\left(d\left(a, R^{-}\right)\right.$.

Theorem I.9.2 ([12], Theorem 43.2): $\quad$ Let $f \in \mathcal{M}(\mathbb{K}),\left(\right.$ resp. $f \in \mathcal{M}\left(d\left(0, R^{-}\right)\right)$ ) and let $g \in \mathcal{M}_{f}(\mathbb{K})$, (resp.g $\in \mathcal{M}_{f}\left(d\left(0, R^{-}\right)\right)$). Then $T(r, f g)=T(r, f)+$ $o(T(r, f))$ and $T\left(r, \frac{f}{g}\right)=T(r, f)+o(T(r, f))$

Theorem I.9.3 ([12], Theorem 43.3): Let $f \in \mathcal{A}(\mathbb{K})$ (resp. let $a \in \mathbb{K}$ and $r>0$ and let $f \in \mathcal{A}_{u}\left(d\left(a, r^{-}\right)\right)$). Let $g, h \in \mathcal{A}_{f}(\mathbb{K})$ (resp. let $g, h \in \mathcal{A}_{f}\left(d\left(a, r^{-}\right)\right.$) with $g$ and $h$ not identically zero. If gh belongs to $\mathcal{A}_{f}(\mathbb{K})$ (resp. to $\mathcal{A}_{f}\left(d\left(a, r^{-}\right)\right)$), then so do $g$ and $h$.

The following theorem is known as the Second Main Theorem on three small functions and is proven in the same way as in $\mathbb{C}$ :

Theorem I.9.4 ([12], Theorem 43.10): Let $f \in \mathcal{M}(\mathbb{K})\left(\right.$ resp. $f \in \mathcal{M}_{u}\left(d\left(0, R^{-}\right)\right)$) and let $w_{1}, w_{2}, w_{3} \in \mathcal{M}_{f}(\mathbb{K})$ (resp. $w_{1}, w_{2}, w_{3} \in \mathcal{M}_{f}\left(d\left(0, R^{-}\right)\right)$) be pairwise distinct. Then $T(r, f) \leq \sum_{j=1}^{3} \bar{Z}\left(r, f-w_{j}\right)+o(T(r, f))$.

Theorem 19.5 closely derives from Theorem 19.4:

Theorem I.9.5 ([12], Theorem 43.11): Let $f \in \mathcal{M}(\mathbb{K})$ (resp. $f \in \mathcal{M}_{u}\left(d\left(0, R^{-}\right)\right)$) and let $w_{1}, w_{2} \in \mathcal{M}_{f}(\mathbb{K})$ (resp. $w_{1}, w_{2} \in \mathcal{M}_{f}\left(d\left(0, R^{-}\right)\right)$) be distinct. Then $\left.T(r, f) \leq \bar{Z}\left(r, f-w_{1}\right)+\bar{Z}\left(r, f-w_{2}\right)+\bar{N}(r, f)+o(T(r, f))\right)$.

Next, by setting $g=f-w_{1}$ and $w=w_{1}+w_{2}$, we can write this Corollary: 
Corollary I.9.5.a: Let $g \in \mathcal{M}(\mathbb{K})$ (resp. $g \in \mathcal{M}_{u}\left(d\left(0, R^{-}\right)\right)$) and let $w \in \mathcal{M}_{g}(\mathbb{K})$ (resp. $w \in \mathcal{M}_{g}\left(d\left(0, R^{-}\right)\right)$). Then $T(r, g) \leq \bar{Z}(r, g)+\bar{Z}(r, g-w)+\bar{N}(r, g)+$ $o(T(r, g)))$.

Corollary I.9.5.b: Let $f \in \mathcal{A}(\mathbb{K})$ (resp. $f \in \mathcal{A}_{u}\left(d\left(0, R^{-}\right)\right.$)) and let $w_{1}, w_{2} \in$ $\mathcal{A}_{f}(\mathbb{K})$ (resp. $w_{1}, w_{2} \in \mathcal{A}_{f}\left(d\left(0, R^{-}\right)\right)$) be distinct. Then

$$
T(r, f) \leq \bar{Z}\left(r, f-w_{1}\right)+\bar{Z}\left(r, f-w_{2}\right)+o(T(r, f)) .
$$

Corollary I.9.5.c: Let $f \in \mathcal{A}(\mathbb{K})$ (resp. $f \in \mathcal{A}_{u}\left(d\left(0, R^{-}\right)\right.$)) and let $w \in \mathcal{A}_{f}(\mathbb{K})$ $\left(\right.$ resp. $u \in \mathcal{A}_{f}\left(d\left(0, R^{-}\right)\right)$.) Then $\left.T(r, f) \leq \bar{Z}(r, f)+\bar{Z}(r, f-w)+o(T(r, f))\right)$.

Here is now an application of that theory:

Theorem I.9.6 ([12], Theorem 43.15): Let $h, w \in \mathcal{A}_{b}\left(d\left(a, R^{-}\right)\right)$and let $m, n \in \mathbb{N}^{*}$ be such that $\min (m, n) \geq 2, \max (m, n) \geq 3$. Then the functional equation

$$
(\mathcal{E}) \quad(g(x))^{n}=h(x)(f(x))^{m}+w(x)
$$

has no solution in $\mathcal{A}_{u}\left(d\left(a, R^{-}\right)\right)$.

\section{Nevanlinna Theory out of a hole}

\section{II.1. Definitions}

We will now show that another Nevanlinna Theory exists for meromorphic functions defined not in an "open" disk, but on the contrary in the complement of such a disk.

Recently, M.O. Hanyak and A.A. Kondratyuk constructed a Nevanlinna theory for meromorphic functions in a punctured complex plane, i.e. in the set of the form $\mathbb{C} \backslash\left\{a_{1}, \ldots, a_{m}\right\}$, where we understand that the meromorphic functions can admit essential singularities at $a_{1}, \ldots, a_{m}[17]$. Here we consider the situation in our field $\mathbb{K}$ of characteristic 0 .

So, we mean to construct a Nevanlinna theory for meromorphic functions in the complement of an open disk thanks to the use of specific properties of the Analytic Elements on infraconnected subsets of $\mathbb{K}$ and particularly the Motzkin Factorization [22].

Once the Nevanlinna Theory is established for such functions, we can apply it to obtain results on uniqueness, Picard's values and branched values as it was done in similar problems.

Throughout that study, we fix a number $R>0$ and denote by $I$ the interval $\left[R,+\infty\left[\right.\right.$. Next, we denote by $S$ the disk $d\left(0, R^{-}\right)$and put $D=\mathbb{K} \backslash S$.

Recall that we denote by $\mathcal{A}(D)$ the $\mathbb{K}$-algebra of Laurent series converging in $D$. Similarly, we will denote by $\mathcal{M}(D)$ the field of fractions of $\mathcal{A}(D)$ that we will call field of meromorphic functions in $D$. 
Given $f \in \mathcal{M}(D)$, for $r>R$, we will denote by $Z_{R}(r, f)$ the counting function of zeros of $f$ between $R$ and $r$, i.e. if $\alpha_{1}, \ldots, \alpha_{m}$ are the distinct zeros of $f$ in $\Delta(0, R, r)$, with respective multiplicity $u_{j}, 1 \leq j \leq m$, then

$Z_{R}(r, f)=\sum_{j=1}^{m} u_{j}\left(\log (r)-\log \left(\left|\alpha_{j}\right|\right)\right)$. Similarly, we denote by $N_{R}(r, f)$ the counting function of poles of $f$ between $R$ and $r$, i.e. if $\beta_{1}, \ldots, \beta_{n}$ are the distinct poles of $f$ in $\Delta(0, R, r)$, with respective multiplicity $v_{j}, 1 \leq j \leq m$, then

$N_{R}(r, f)=\sum_{j=1}^{n} v_{j}\left(\log (r)-\log \left(\left|\beta_{j}\right|\right)\right)$. Finally we put

$T_{R}(r, f)=\max \left(Z_{R}(r, f), N_{R}(r, f)\right)$.

Next, we denote by $\bar{Z}_{R}(r, f)$ the counting function of zeros without counting multiplicity: if $\alpha_{1}, \ldots, \alpha_{m}$ are the distinct zeros of $f$ in $\Delta(0, R, r)$, then we put $\bar{Z}_{R}(r, f)=\sum_{j=1}^{m} \log (r)-\log \left(\left|\alpha_{j}\right|\right)$.

Similarly, we denote by $\bar{N}_{R}(r, f)$ the counting function of poles without counting multiplicity: if $\beta_{1}, \ldots, \beta_{n}$ are the distinct poles of $f$ in $\Delta(0, R, r)$, then we put $\bar{N}_{R}(r, f)=\sum_{j=1}^{n} \log (r)-\log \left(\left|\beta_{j}\right|\right)$.

Finally, putting $W=\left\{a_{1}, \ldots, a_{q}\right\}$, we denote by $Z_{R}^{W}\left(r, f^{\prime}\right)$ the counting function of zeros of $f^{\prime}$ on points where $f(x) \notin W$.

By Theorem I.5.3 we can derive Theorem II.1.1:

Theorem II.1.1: Let $f \in \mathcal{M}(D)$. We can write $f$ in a unique way in the form $f^{S} f^{0}$ with $f^{S} \in H(D)$ a Motzkin factor associated to $S$ and $f^{0} \in \mathcal{M}(\mathbb{K})$, having no zero and no pole in $S$.

Proof. $\quad$ Suppose first $f \in \mathcal{A}(D)$ and take $V>R$. Then as a quasi-invertible element of $H(\Delta(0, R, V))$ [9], [12], by Theorem I.5.3, $f$ admits a factorization in the form $f^{S} f^{0}$ where $f^{S}$ is a Motzkin factor and $f^{0}$ belongs to $H(d(0, V))$ and has no zero in $S$. Moreover by Theorem I.5.2, $f^{S}$ does not depend on $V$. Consequently, since $f^{S}$ is obviously invertible in $\mathcal{A}(D)$, we can factorize $f \in \mathcal{A}(D)$ in the form $f^{S} f^{0}$ where $f^{0}$ belongs to $\mathcal{A}(\mathbb{K})$ and has no zero in $S$.

Consider now the general case: $f=\frac{g}{h}$ with $g, h \in \mathcal{A}(D)$. Then we can write $g=g^{S} g^{0}, h=h^{S} h^{0}$, hence $f=\left(\frac{g^{S}}{h^{S}}\right)\left(\frac{g^{0}}{h^{0}}\right)$. Then we check that this is the factorization announced in the statement: $f^{S}=\frac{g^{S}}{h^{S}}$ and $f^{0}=\frac{g^{0}}{h^{0}}$. The uniqueness also comes from Theorem I.5.3.

The following lemmas are immediate:

Lemma II.1.2: Let $f, g \in \mathcal{A}(D)$. If $|f|(r)>|g|(r)$, then $|f+g|(r)=|f|(r)$. 
Lemma II.1.3: The set of Motzkin factors associated to $S$ makes a multiplicative group. Let $f, g \in \mathcal{M}(D)$. Then $(f g)^{S}=\left(f^{S}\right)\left(g^{S}\right),\left(\frac{1}{f}\right)^{S}=\frac{1}{f^{S}},(f g)^{0}=$ $\left(f^{0}\right)\left(g^{0}\right),\left(\frac{1}{f}\right)^{0}=\frac{1}{f^{0}}$ and $m(f g, S)=m(f, S)+m(g, S), m\left(\frac{1}{f}, S\right)=-m(f, S)$.

Proof. We have $f=f^{S} f^{0}$. Since $f^{S}$ has no zero and no pole in $D$, by Lemma I.1 it satisfies $\left.\left|f^{S}\right|(r)\right)=r^{m(f, S)}$ for all $r \in I$, hence $\log \left(\left|f^{S}\right|(r)\right)$ $\log \left(\left|f^{S}\right|(R)\right)=m(f, S)(\log r-\log R)(r \in I)$. Next, since $f^{0}$ has no zero and no pole in $S$, we have $\log \left(\left|f^{0}\right|(r)\right)-\log \left(\left|f^{0}\right|(R)\right)=Z_{R}\left(r, f^{0}\right)-N_{R}\left(r, f^{0}\right)(r \in I)$. Therefore the statement is clear.

Notation: We will denote by $\mathcal{M}^{*}(D)$ the set of $f \in \mathcal{M}(D)$ such that $f^{0} \notin \mathbb{K}(x)$ i.e. the set of $f \in \mathcal{M}(D)$ admitting at least infinitely many zeros in $D$ or infinitely many poles in $D$. Similarly, we will denote by $\mathcal{A}^{*}(D)$ the set of $f \in \mathcal{A}(D)$ such that $f^{0} \notin \mathbb{K}[x]$ i.e. the set of $f$ admitting infinitely many zeros in $D$. Next, we set $\mathcal{M}^{0}(D)=\mathcal{M}(D) \backslash \mathcal{M}^{*}(D)$ and $\mathcal{A}^{0}(D)=\mathcal{A}(D) \backslash \mathcal{A}^{*}(D)$.

Theorem II.1.4 is similar to Theorem I.7.2:

Theorem II.1.4: Let $f \in \mathcal{M}(D)$. Then

$$
\log (|f|(r))-\log (|f|(R))=Z_{R}(r, f)-N_{R}(r, f)+m(f, S)(\log r-\log R), \quad(r \in I) .
$$

Proof. By Theorem T.I.1, we have $f=f^{S} f^{0}$. Since $f^{S}$ has no zero and no pole in $D$, by Lemma I.1 it satisfies $\left.\left|f^{S}\right|(r)\right)=r^{m(f, S)}$ for all $r \in I$, hence $\log \left(\left|f^{S}\right|(r)\right)-\log \left(\left|f^{S}\right|(R)\right)=m(f, S)(\log r-\log R)(r \in I)$. Next, since $f^{0}$ has no zero and no pole in $S$, we have $\log \left(\left|f^{0}\right|(r)\right)-\log \left(\left|f^{0}\right|(R)\right)=Z_{R}\left(r, f^{0}\right)-N_{R}\left(r, f^{0}\right)(r \in$ $I)$. Therefore the statement is clear.

Corollary II.1.4.a: Let $f \in \mathcal{M}(D)$. Then $T_{R}(r, f)$ is identically zero if and only if $f f^{0}$ is a constant.

Corollary II.1.4.b: Let $f \in \mathcal{A}(D)$ and let $\phi \in H_{0}(D)$. Then $Z_{R}(r, f+\phi)=$ $Z_{R}(r, f)+O(\log (r))(r \in I)$.

Corollary II.1.4.c: Let $f, g \in \mathcal{A}(D)$ satisfy $\log (|f|(r)) \leq \log (|g|(r)) \forall r \geq R$ $(r \in I)$. Then $Z_{R}(r, f) \leq Z_{R}(r, g)+(m(g, S)-m(f, S))(\log (r)-\log (R)), \quad(r \in I)$.

We can now characterize the set $\mathcal{M}^{*}(D)$ :

Theorem II.1.5: Let $f \in \mathcal{A}(D)$. Then $Z_{R}\left(r, f^{\prime}\right) \leq Z_{R}(r, f)+O(\log (r))(r \in I)$.

Proof. Indeed, by Theorem 18.1 in [12], we have $\left|f^{\prime}\right|(r) \leq|f|(r)$ and hence the conclusion comes from Theorem II.1.4.

Corollary II.1.5.1: Let $f \in \mathcal{A}(D)$. Then $Z_{R}\left(\frac{f^{\prime}}{f}\right)-N_{R}\left(r, \frac{f^{\prime}}{f}\right) \leq O(\log (r))$.

Theorem II.1.6: Let $f \in \mathcal{M}(D)$. The three following statements are equivalent: i) $\lim _{r \rightarrow+\infty} \frac{T_{R}(r, f)}{\log (r)}=+\infty(r \in I)$, 
ii) $\frac{T_{R}(r, f)}{\log (r)}$ is unbounded,

iii) $f$ belongs to $\mathcal{M}^{*}(D)$.

Proof. Consider an increasing sequence $\left(u_{n}\right)_{n \in \mathbb{N}}$ in $\mathbb{R}_{+}$such that $\lim _{n \rightarrow+\infty} u_{n}=+\infty$ and let $\left(k_{n}\right)_{n \in \mathbb{N}}$ be a sequence of $\mathbb{N}^{*}$. Clearly, we have

$$
\lim _{r \rightarrow+\infty} \frac{\sum_{u_{n} \leq r} k_{n}\left(\log (r)-\log \left(u_{n}\right)\right)}{\log (r)}=+\infty .
$$

Consequently, if a function $f \in \mathcal{M}^{*}(D)$ has infinitely many zeros (resp. infinitely many poles in $D$ ) then $\lim _{n \rightarrow+\infty} \frac{Z_{R}(r, f)}{\log (r)}=+\infty$ (resp. $\lim _{n \rightarrow+\infty} \frac{N_{R}(r, f)}{\log (r)}=+\infty$ ) hence in both cases, $\lim _{n \rightarrow+\infty} \frac{T_{R}(r, f)}{\log (r)}=+\infty$. Conversely, if $f$ has finitely many zeros and finitely many poles in $D$, then we check that $\lim _{n \rightarrow+\infty} \frac{T_{R}(r, f)}{\log (r)}<+\infty$. Thus the equivalence of the three statements is clear.

Operations on $\mathcal{M}(D)$ work almost like for meromorphic functions in the whole field.

Theorem II.1.7: Let $f, g \in \mathcal{M}(D)$. Then for any $b \in \mathbb{K}$ we have

$T_{R}(r, f+b)=T_{R}(r, f)+O(\log (r)),(r \in I)$

$T_{R}(r, f . g) \leq T_{R}(r, f)+T_{R}(r, g)+O(\log (r)),(r \in I)$,

$T_{R}\left(r, \frac{1}{f}\right)=T_{R}(r, f),(r \in I)$,

$T_{R}(r, f+g) \leq T_{R}(r, f)+T_{R}(r, g)+O(\log (r)),(r \in I)$

and $T_{R}\left(r, f^{n}\right)=n T_{R}(r, f),(r \in I)$.

Let $h$ be a Moebius function. Then

$T_{R}(r, h \circ f)=T_{R}(r, f)+O(\log (r)),(r \in I)$.

Moreover, if both $f$ and $g$ belong to $\mathcal{A}(D)$, then

$$
T_{R}(r, f+g) \leq \max \left(T_{R}(r, f), T_{R}(r, g)\right)+O(\log (r)), \quad(r \in I)
$$

and $T_{R}(r, f g)=T_{R}(r, f)+T_{R}(r, g),(r \in I)$.

Particularly, if $f \in \mathcal{A}^{*}(D)$, then $T_{R}(r, f+b)=T_{R}(r, f)+O(1),(r \in I)$.

Given a polynomial $P$ of degree $q$, then $T_{R}(r, P \circ f)=q T_{R}(r, f)$.

Corollary II.1.7.a: Let $f, g \in \mathcal{M}^{0}(D)$. Then

$T_{R}\left(r, \frac{f}{g}\right) \geq T_{R}(r, f)-T_{R}(r, g)(r \in I)$ and $T_{R}\left(r, \frac{f}{g}\right) \geq T_{R}(r, g)-T_{R}(r, f)(r \in I)$.

By Theorems II.1.6 and II.1.7, now we have this immediate corollary:

Corollary II.1.7.b: $\mathcal{M}^{0}(D)$ is a subfield of $\mathcal{M}(D)$.

Theorem II.1.8: Every $f \in \mathcal{M}^{*}(D)$ is transcendental over $\mathcal{M}^{0}(D)$.

Proof. Consider a polynomial $P(Y)=\sum_{j=0}^{n} a_{j} Y^{j} \in \mathcal{M}^{0}(D)[Y]$ with 
$a_{n}=1$. Let $f \in \mathcal{M}^{*}(D)$ and suppose that $P(f)=0$. Then $f^{n}=-\sum_{j=0}^{n-1} a_{j} f^{j}$. Set $\Xi=\sum_{j=0}^{n-1} a_{j} f^{j}$ and $f=f^{0} \frac{g}{h}$ with $g, h \in \mathcal{A}(D)$ having no zero in $S$. Then $\Xi=\frac{\sum_{j=0}^{n-1} a_{j} g^{j} h^{n-1-j}}{h^{n-1}}$.

Since $\sum_{j=0}^{n-1} a_{j} g^{j} h^{n-1-j}$ belongs to $\mathcal{A}(D)$, by Theorem II.3 we have

$$
T_{R}\left(r, \sum_{j=0}^{n-1} a_{j} g^{j} h^{n-1-j}\right) \leq(n-1) T_{R}(r, f)+O(\log (r)),(r \in I)
$$

and of course $T_{R}\left(r, h^{n-1}\right) \leq(n-1) T_{R}(r, f),(r \in I)$. Consequently

$$
T_{R}(r, \Xi) \leq(n-1) T_{R}(r, f)+O(\log (r),(r \in I) .
$$

But on the other hand, by Theorem II.3, $T_{R}\left(r, f^{n}\right)=n T_{R}(r, f)$. Therefore we should have $n T_{R}(r, f) \leq(n-1) T_{R}(r, f)+O(\log (r),(r \in I)$, which is impossible by Theorem II.2 because $f$ belongs to $\mathcal{M}^{*}(D)$. Consequently, the equality $P(f)=0$ is impossible, which proves that $f$ is transcendental over $\mathcal{M}^{0}(D)$.

In Theorem II.1.9 we find results already stated about derivatives, for functions in the whole field or in an open disk. The difference only holds in the rest:

Theorem II.1.9: Let $f \in \mathcal{M}(D)$. Then

$$
N_{R}\left(r, f^{(k)}\right)=N_{R}(r, f)+k \bar{N}_{R}(r, f),(r \in I)
$$

and

$$
Z_{R}\left(r, f^{(k)}\right) \leq Z_{R}(r, f)+k \bar{N}_{R}(r, f)+O(\log (r)),(r \in I)
$$

Proof. The inequality $N_{R}\left(r, f^{(k)}\right)=N_{R}(r, f)+k \bar{N}_{R}(r, f)+O(1)$ is obvious. Next consider $f$ in the form $\frac{g}{h}$ with $g, h \in \mathcal{A}(\mathbb{K})$. Recall that we can write $h$ in the form $\bar{h} \widetilde{h}$ with $\bar{h}$ and $\widetilde{h}$ in $\mathcal{A}(\mathbb{K})$, each zero of $\bar{h}$ being of order one and all zeros of $h$ being a zero of $\bar{h}$. So, $h^{\prime}$ is of the form $\widetilde{h} \widehat{h}$ where $\widehat{h}$ belong to $\mathcal{A}(\mathbb{K})$ and none of the zeros of $\widehat{h}$ is a zero of $h$. Then $f^{\prime}$ is of the form $\frac{g^{\prime} \bar{h}-g \widehat{h}}{h \bar{h}}$. So, $Z_{R}\left(r, f^{\prime}\right) \leq Z_{R}\left(r, g^{\prime} \bar{h}-g \widehat{h}\right)$ and hence, $Z_{R}\left(r, f^{\prime}\right) \leq \max \left(Z_{R}\left(r, g^{\prime} \bar{h}\right), Z_{R}(r, g \widehat{h})\right.$.

On the other hand, by Theorem II.1.5, $Z_{R}\left(r, g^{\prime}\right) \leq Z_{R}(r, g)+O(\log r)$. Obviously, $Z_{R}(r, \bar{h}) \leq \bar{Z}_{R}(r, h)=\bar{N}_{R}(r, \ell)=\bar{N}_{R}(r, f)$ hence $Z_{R}\left(r,\left(r, g^{\prime} \bar{h}\right) \leq Z_{R}(r, f)+\right.$ $\bar{N}_{R}(r, f)$.

Now, let us estimate $Z_{R}(r, \widehat{h})$. Since $\log \left(\left|h^{\prime}\right|(r)\right) \leq \log (|h|(r))-\log r$, we have

$$
Z_{R}\left(r, h^{\prime}\right) \leq Z_{R}(r, h)+O(\log (r)) .
$$

But since $h^{\prime}=\widehat{h} \widetilde{h}$, we have

$$
Z_{R}(r, \widehat{h})=Z_{R}\left(r, h^{\prime}\right)-Z_{R}(r, \widetilde{h}) \leq Z_{R}(r, h)-Z_{R}(r, \widetilde{h})+O(\log (r))
$$




$$
=Z_{R}(r, \bar{h})+O(\log (r))=\bar{N}_{R}(r, f)+O(\log (r)) .
$$

Consequently,

$$
Z_{R}(r, g \widehat{h}) \leq Z_{R}(r, g)+\bar{N}_{R}(r, f)+O(\log (r))=Z_{R}(r, f)+\bar{N}_{R}(r, f)+O(\log (r)) .
$$

Thus, by (1), we have proven the claim when $k=1$ and then it is immediately derived by induction on $k$.

We can now define small functions in the same way as on the full field $\mathbb{K}$ or as on the disk $d\left(0, R^{-}\right)$

Definitions and notation: For each $f \in \mathcal{M}(D)$ we denote by $\mathcal{M}_{f}(D)$ the set of functions $h \in \mathcal{M}(D)$ such that $T_{R}(r, h)=o\left(T_{R}(r, f)\right)$ when $r$ tends to $+\infty$. Similarly, if $f \in \mathcal{A}(D)$ we denote by $\mathcal{A}_{f}(D)$ the set $\mathcal{M}_{f}(D) \cap \mathcal{A}(D)$.

The elements of $\mathcal{M}_{f}(D)$ are called small meromorphic functions with respect to $f$, small functions in brief. Similarly, if $f \in \mathcal{A}(D)$ the elements of $\mathcal{A}_{f}(D)$ are called small analytic functions with respect to $f$, small functions in brief.

A function $w \in \mathcal{M}_{f}(D)$ will be called an exceptional small function with respect to $f$ if $f-w$ has no zero in $D$ and it will be called a quasi-exceptional small function with respect to $f$ if $f-w$ has finitely many zeros in $D$.

Theorem II.1.10: $\mathcal{A}_{f}(D)$ is a $\mathbb{K}$-subalgebra of $\mathcal{A}(D), \mathcal{M}_{f}(D)$ is a subfield of $\mathcal{M}(D)$.

Let $f \in \mathcal{M}(D)$ and let $g \in \mathcal{M}_{f}(D)$. Then $T_{R}(r, f g)=T_{R}(r, f)+o\left(T_{R}(r, f)\right)$ $(r \in I)$ and $T_{R}\left(r, \frac{f}{g}\right)=T_{R}(r, f)+o\left(T_{R}(r, f)\right)(r \in I)$.

Let $g, h \in \mathcal{A}(D)$ with $g$ and $h$ not identically zero. If gh belongs to $\mathcal{A}_{f}(D)$ then so $d o g$ and $h$.

Theorem II.1.11: Let $f \in \mathcal{M}^{*}(D)$. There exists at most one function $w \in$ $\mathcal{M}_{f}(D)$, such that $f-w$ have finitely many zeros in $D$. Moreover, if $f$ has finitely many poles, then there exists no function $w \in \mathcal{M}_{f}(D)$, such that $f-w$ have finitely many zeros in $D$.

Proof. Suppose that $f$ admits two distinct quasi-exceptional small functions. Without loss of generality we may assume that these functions are 0 and $w \in$ $\mathcal{M}_{f}(D)$. Let $g=f-w$, let $f^{S}$ be the Motzkin factor of $f$ associated to $S$ and let $g^{S}$ be the Motzkin factor of $g$ associated to $S$. Then $f$ is of the form $f^{S} \frac{P}{h}$ with $h \in \mathcal{A}^{*}(D)$ and $P \in \mathbb{K}[x]$ having all its zeros in $D$ and $g$ is of the form $g^{S} \frac{Q}{l}$ with $l \in \mathcal{A}^{*}(D)$ and $Q \in \mathbb{K}[x]$ having all its zeros in $D$. Consequently, we have $h g^{S} Q-l f^{S} P=h l w$. Now, by Theorem II.1.7, we have

$$
\begin{gathered}
T_{R}\left(r, h g^{S} Q-l f^{S} P\right) \leq \max \left(T_{R}\left(r, h g^{S} Q\right), T_{R}\left(r, l f^{S} P\right)\right)+O(\log (r)), \\
\leq\left(T_{R}(r, h), T_{R}(r, l)+O(\log (r)),(r \in I),\right.
\end{gathered}
$$

hence

$$
T_{R}(r, h l w) \leq \max \left(\left(T_{R}(r, h), T_{R}(r, l)\right)+O(\log (r)), \quad(r \in I) .\right.
$$

Next, by Theorem II.1.7 and Corollary II.1.7.a we have

$$
T_{R}(r, w l h) \geq T_{R}(r, h)+T_{R}(r, l)-T_{R}(r, w) .
$$


Now, since $f=f^{S} \frac{P}{h}$, clearly $T_{R}(r, f)=T_{R}(r, h)+O(\log (r))$ and similarly, $T_{R}(r, g)=$ $T_{R}(r, l)+O(\log (r))$. But since $T_{R}(r, w)=o\left(T_{R}(r, f)\right.$, by Theorem II.1.10 we can check that $T_{R}(r, g)=T_{R}(r, f)+o\left(T_{R}(r, f)\right)$. Consequently, $T_{R}(r, l)=T_{R}(r, h)+$ $o\left(T_{R}(r, h)\right)$. Therefore by $(1)$ we have $T_{R}(r, h l w) \leq\left(T_{R}(r, h)+o\left(T_{R}(r, h), \quad(r \in I)\right.\right.$ and by (2) we obtain $T_{R}(r, w l h) \geq 2 T_{R}(r, h)+o\left(T_{R}(r, f)\right)(r \in I)$, a contradiction. This proves that $f$ cannot have two small functions $w \in \mathcal{M}_{f}(D)$ such that $f-w$ have finitely many zeros.

Suppose now that $f \in \mathcal{M}^{*}(D)$ has finitely many poles and admits a quasiexceptional small function $w$. Set $g=f-w$. Then $g$ is of the form $g^{S}\left(\frac{P}{h}\right)$ where $P$ is a polynomial whose zeros lie in $D$ and $h$ belongs to $\mathcal{A}(D)$ and it admits for zeros the poles of $f$ and those of $w$. Consequently, $h$ belongs to $\mathcal{A}_{f}(D)$. Therefore we can check that $T_{R}(r, g)=o\left(T_{R}(r, f)\right.$. But by Corollary II.1.7.a we have $T_{R}(r, g) \geq$ $T_{R}(r, f)-T_{R}(r, w)=T_{R}(r, f)+o\left(T_{R}(r, f)\right)$, a contradiction.

Corollary II.1.11.a: Let $f \in \mathcal{A}^{*}(D)$. Then $f$ has no quasi-exceptional small function.

Lemma II.1.12 will be necessary in the proof of Theorem II.1.13. It looks like Corollary 2.15 in [5]:

Lemma II.1.12: $\quad$ Let $f \in \mathcal{M}(D)$. Suppose that there exists $\xi \in \mathbb{K}$ and a sequence of intervals $J_{n}=\left[u_{n}, v_{n}\right]$ such that $u_{n}<v_{n}<u_{n+1}, \lim _{n \rightarrow+\infty} u_{n}=+\infty$ and $\lim _{n \rightarrow+\infty}\left[\inf _{r \in J_{n}} \frac{T_{R}(r, f)-Z_{R}(r, f-\xi)}{\log (r)}\right]=+\infty$.

Let $\tau \in \mathbb{K} \tau \neq \xi$. Then $\left.Z_{R}(r, f-\tau)=T_{R}(r, f)+O(\log (r))\right) \forall r \in J_{n}$ when $n$ is big enough.

Proof. Without loss of generality, we can obviously suppose that $\xi=0$. By Theorem II.1.1, $f$ is of the form $f^{S} f^{0}$ and $f^{0}$ is of the form $\frac{g}{h}$ with $g, h \in \mathcal{A}(D)$, having no zero in $S$. Set $w=f^{S}$. Thus we have

$$
\lim _{n \rightarrow+\infty}\left[\inf _{r \in J_{n}} \frac{Z_{R}(r, h)-Z_{R}(r, g)}{\log (r)}\right]=+\infty .
$$

Consequently, by Theorem II.1.4, we have

$$
\lim _{n \rightarrow+\infty}\left[\inf _{r \in J_{n}} \frac{\log (|h|(r)-\log (|g|(r)}{\log (r)}\right]=+\infty .
$$

Consider now $f-\tau$. We have $f-\tau=\frac{w g-\tau h}{h}$, hence

$$
\log (|f|(r))=\log (|w g-\tau h|(r)-\log (|h|(r)) .
$$

But by $(\mathrm{A})$, we have $\log (|\tau h|(r))>\log (|w g|(r))$ because $\log (|w|(r)=O(\log (r))$, therefore by Lemma II.1.2 $\log (|w g-\tau h|(r))=\log (|\tau h|(r)) \forall r \in J_{n}$ when $n$ is big enough and hence

$$
\lim _{n \rightarrow+\infty}\left[\sup _{r \in J_{n}} \frac{\log (|\tau h-w g|(r)-\log (|h|(r)}{\log (r)}\right]=0 .
$$


Consequently, by (2) and by Theorem II.1.4,

$$
\lim _{n \rightarrow+\infty}\left[\sup _{r \in J_{n}} \frac{Z_{R}(r, \tau h-w g)-Z_{R}(r, h)}{\log (r)}\right]=0
$$

i.e.

$$
\lim _{n \rightarrow+\infty}\left[\sup _{r \in J_{n}} \frac{Z_{R}(r, f-\tau)-T_{R}(r, f)}{\log (r)}\right]=0
$$

which proves the claim.

Similarly to the reasonning when we considered meromorphic functions in $\mathbb{K}$ or inside a disk $d\left(a, R^{-}\right)$, the Nevanlinna second Main Theorem is based on the following theorem:

Theorem II.1.13: $\quad$ Let $f \in \mathcal{M}(D)$ and let $a_{1}, \ldots, a_{q} \in \mathbb{K}$ be distinct. Then

$$
(q-1) T_{R}(r, f) \leq \max _{1 \leq k \leq q}\left(\sum_{j=1, j \neq k}^{q} Z_{R}\left(r, f-a_{j}\right)\right)+O(\log (r))(r \in I) .
$$

Proof. Suppose Theorem II.1.13 is wrong. Thus, there exists $f \in \mathcal{M}(D)$ and $a_{1}, \ldots, a_{q} \in \mathbb{K}$ such that

$$
(q-1) T_{R}(r, f)-\max _{1 \leq k \leq q}\left(\sum_{j=1, j \neq k}^{q} Z_{R}\left(r, f-a_{j}\right)\right)
$$

admits no superior bound in $] 0,+\infty\left[\right.$. So, there exists a sequence of intervals $J_{s}=$ $\left[w_{s}, y_{s}\right]$ such that $w_{s}<y_{s}<w_{s+1}, \lim _{s \rightarrow+\infty} w_{s}=+\infty$ and two distinct indices $m \leq q$ and $t \leq q$ such that

$$
\lim _{s \rightarrow+\infty}\left[\inf _{r \in J_{s}} \frac{\left(T_{R}(r, f)-Z_{R}\left(r, f-a_{m}\right)\right)}{\log (r)}\right]=+\infty
$$

and

$$
\lim _{s \rightarrow+\infty}\left[\inf _{r \in J_{s}} \frac{\left(T_{R}(r, f)-Z_{R}\left(r, f-a_{t}\right)\right)}{\log (r)}\right]=+\infty
$$

But by Lemma II.1, that is impossible. This ends the proof of Theorem II.1.13.

Corollary II.1.13.a: Let $f \in \mathcal{M}(\mathbb{K})$ and let $a_{1}, \ldots, a_{q} \in \mathbb{K}$ be distinct. Then $(q-1) T_{R}(r, f) \leq \sum_{j=1}^{q} Z_{R}\left(r, f-a_{j}\right)+O(\log (r))(r \in I)$

We can now state and prove the Second Main Theorem for $\mathcal{M}(D)$.

Theorem II.1.14: Let $f \in \mathcal{M}(D)$, let $\alpha_{1}, \ldots, \alpha_{q} \in \mathbb{K}$, with $q \geq 2$ and let $W=\left\{\alpha_{1}, \ldots, \alpha_{q}\right\}$. Then $(q-1) T_{R}(r, f) \leq$

$$
\sum_{j=1}^{q} \bar{Z}_{R}\left(r, f-\alpha_{j}\right)+Z_{R}\left(r, f^{\prime}\right)-Z_{R}^{W}\left(r, f^{\prime}\right)+O(\log (r)) \quad(r \in I) .
$$

Moreover, if $f$ belongs to $\mathcal{A}(D)$ then $q T_{R}(r, f) \leq$

$$
\leq \sum_{j=1}^{q} \bar{Z}_{R}\left(r, f-\alpha_{j}\right)+Z_{R}\left(r, f^{\prime}\right)-Z_{R}^{W}\left(r, f^{\prime}\right)+O(\log (r))(r \in I) .
$$




\section{Theorem II.1.15 (Second Main Theorem):}

Let $f \in \mathcal{M}(D)$, let $\alpha_{1}, \ldots, \alpha_{q} \in \mathbb{K}$, with $q \geq 2$ and let $W=\left\{\alpha_{1}, \ldots, \alpha_{q}\right\}$. Then $(q-1) T_{R}(r, f) \leq$

$$
\sum_{j=1}^{q} \bar{Z}_{R}\left(r, f-\alpha_{j}\right)+\bar{N}_{R}(r, f)-Z_{R}^{W}\left(r, f^{\prime}\right)+O(\log (r))(r \in I) .
$$

Corollary II.1.15.a: Let $f \in \mathcal{M}(D)$, let $\alpha_{1}, \ldots, \alpha_{q} \in \mathbb{K}$, with $q \geq 2$ and let $W=\left\{\alpha_{1}, \ldots, \alpha_{q}\right\}$. Then

$$
\begin{gathered}
\sum_{j=1}^{q}\left(Z_{R}\left(r, f-\alpha_{j}\right)-\bar{Z}_{R}\left(r, f-\alpha_{j}\right)\right) \\
\leq T_{R}(r, f)+\bar{N}_{R}(r, f)-Z_{R}^{W}\left(r, f^{\prime}\right)+O(\log (r)) \quad(r \in I) .
\end{gathered}
$$

Proof. of Theorems II.1.14 and II.1.15: $\quad$ By Theorem II.1.13 there exists a constant $B>0$ and for each $r>R$ there exists $k(r) \in \mathbb{N}, k(r) \leq q$, such that

$$
(q-1) T_{R}(r, f) \leq \sum_{j=1, j \neq k(r)}^{q} Z_{R}\left(r, f-a_{j}\right)+B \log (r)
$$

i.e. $(q-1) T_{R}(r, f) \leq \sum_{j=1}^{q} Z_{R}\left(r, f-a_{j}\right)-Z_{R}\left(r, a_{k(r)}+O(\log (r))\right.$. Now,

$$
\sum_{j=1}^{q} Z_{R}\left(r, f-a_{j}\right)=\sum_{j=1}^{q} \bar{Z}_{R}\left(r, f-a_{j}\right)+Z_{R}\left(r, f^{\prime}\right)-Z_{R}^{W}\left(r, f^{\prime}\right)+B \log (r) .
$$

Consequently,

$$
(q-1) T_{R}(r, f) \leq \sum_{j=1}^{q} \bar{Z}_{R}\left(r, f-a_{j}\right)+Z_{R}\left(r, f^{\prime}\right)-Z_{R}^{W}\left(r, f^{\prime}\right)-Z_{R}\left(r, f-a_{k(r)}\right)+O(\log (r))
$$

and this proves the first claim of Theorem II.1.14.

Particularly, if $f \in \mathcal{A}(D)$ then we have $Z_{R}\left(r, f-a_{j}\right)=T_{R}\left(r, f-a_{j}\right)=T_{R}(r, f)+$ $O(\log (r)) \forall j=1, \ldots, q$, hence $Z_{R}\left(r, f-a_{k(r)}\right)=T_{R}(r, f)+O(\log (r))$ and therefore

$$
q T_{R}(r, f) \leq \sum_{j=1}^{q} \bar{Z}_{R}\left(r, f-a_{j}\right)+Z_{R}\left(r, f^{\prime}\right)-Z_{R}^{W}\left(r, f^{\prime}\right)+O(\log (r)),
$$

which ends the proof of Theorem II.1.14.

Consider now the situation in Theorem II.1.15. By Theorem II.1.13, for each $j=1, \ldots, q$, there exists a constant $B_{j}>0$ such that $Z_{R}\left(r, f^{\prime}\right) \leq Z_{R}\left(r, f-a_{j}\right)+$ $\left.\bar{N}_{R}\left(r, f-a_{j}\right)+B_{j} \log (r)\right)$. Consequently, there exists a constant $C>0$ such that $Z_{R}\left(r, f^{\prime}\right) \leq Z_{R}\left(r, f-a_{k(r)}\right)+\bar{N}_{R}\left(r, f-a_{k(r)}\right)+C \log (r) \forall r>R$.

Therefore, by Relation (1) that remains true in Theorem II.1.15, we can derive $(q-1) T_{R}(r, f) \leq \sum_{j=1}^{q} \bar{Z}_{R}\left(r, f-\alpha_{j}\right)+\bar{N}_{R}(r, f)-Z_{R}^{W}\left(r, f^{\prime}\right)+O(\log (r)) \quad \forall r \in I$. 
We can now find again a Second Main Theorem on Three Small Functions.

Theorem II.1.16: Let $f \in \mathcal{M}^{*}(D)$ and let $w_{1}, w_{2}, w_{3} \in \mathcal{M}_{f}(D)$ be pairwise distinct. Then

$$
T_{R}(r, f) \leq \sum_{j=1}^{3} \bar{Z}_{R}\left(r, f-w_{j}\right)+\sum_{j=1}^{3} T\left(r, w_{j}\right)+O(\log (r))(r \in I) .
$$

ProOF. of Theorem II.1.16: Let $\phi(x)=\frac{\left(f(x)-w_{1}(x)\right)\left(w_{2}(x)-w_{3}(x)\right)}{\left(f(x)-w_{3}(x)\right)\left(w_{2}(x)-w_{1}(x)\right)}$. By Theorem II.12 we have

(1) $T_{R}(r, \phi) \leq \bar{Z}_{R}(r, \phi)+\bar{Z}_{R}(r, \phi-1)+\bar{N}_{R}(r, \phi)+O(\log (r))$.

On the other hand, we have $T_{R}(r, f) \leq T_{R}\left(r, f-w_{j}\right)+T_{R}\left(r, w_{j}\right)(j=1,2,3)$, hence $T_{R}(r, f) \leq T_{R}\left(r, \frac{w_{3}-w_{1}}{f-w_{3}}\right)+o\left(T_{R}(r, f)\right)$, thereby

$T_{R}(r, f) \leq T_{R}\left(r, \frac{w_{3}-w_{1}}{f-w_{3}}+1\right)+o\left(T_{R}(r, f)\right)=T_{R}\left(r, \frac{f-w_{1}}{f-w_{3}}\right)+o\left(T_{R}(r, f)\right)$.

Now, $T_{R}\left(r, \frac{w_{2}-w_{1}}{w_{2}-w_{3}}\right)=o\left(T_{R}(r, f)\right)$. Consequently, by writing $\frac{f-w_{1}}{f-w_{3}}=\left(\frac{w_{2}-w_{1}}{w_{2}-w_{3}}\right) \phi$ we have

$$
T_{R}\left(r, \frac{f-w_{1}}{f-w_{3}}\right) \leq T_{R}(r, \phi)+T_{R}\left(r, \frac{w_{2}-w_{1}}{w_{2}-w_{3}}\right) \leq T_{R}(r, \phi)+o\left(T_{R}(r, f)\right)
$$

and finally $T_{R}(r, f) \leq T_{R}(r, \phi)+o\left(T_{R}(r, f)\right)$. Thus, by (1) we obtain

(2) $T_{R}(r, f) \leq \bar{Z}_{R}(r, \phi)+\bar{Z}_{R}(r, \phi-1)+\bar{N}_{R}(r, \phi)+o\left(T_{R}(r, f)\right)$.

Now, we can check that $\bar{Z}_{R}(r, \phi)+\bar{Z}_{R}(r, \phi-1)+\bar{N}_{R}(r, \phi) \leq \sum_{j=1}^{3} \bar{Z}_{R}\left(r, f-w_{j}\right)+\sum_{1 \leq j<k \leq 3} \bar{Z}_{R}\left(r, w_{k}-w_{j}\right) \leq$ $\leq \sum_{j=1}^{3} \bar{Z}_{R}\left(r, f-w_{j}\right)+o\left(T_{R}(r, f)\right)$ which, by $(2)$, completes the proof.

Corollary II.1.16.a: Let $f \in \mathcal{M}^{*}(D)$ and let $w_{1}, w_{2}, w_{3} \in \mathcal{M}^{0}(D)$ be pairwise distinct. Then

$$
T_{R}(r, f) \leq \sum_{j=1}^{3} \bar{Z}_{R}\left(r, f-w_{j}\right)+O(\log (r))(r \in I)
$$

As an easy consequence, we have

Theorem II.1.17: Let $f \in \mathcal{M}^{*}(D)$ and let $w_{1}, w_{2} \in \mathcal{M}_{f}(D)$ be distinct. Then

$$
\begin{aligned}
& T_{R}(r, f) \leq \bar{Z}_{R}\left(r, f-w_{1}\right)+\bar{Z}_{R}\left(r, f-w_{2}\right)+\bar{N}_{R}(r, f) \\
& +T_{R}\left(r, w_{1}\right)+T_{R}\left(r, w_{2}\right)+O(\log (r))(r \in I) .
\end{aligned}
$$

Corollary II.1.17.a: Let $f \in \mathcal{M}^{*}(D)$ and let $w_{1}, w_{2} \in \mathcal{M}^{0}(D)$ be distinct. Then

$$
T_{R}(r, f) \leq \bar{Z}_{R}\left(r, f-w_{1}\right)+\bar{Z}_{R}\left(r, f-w_{2}\right)+\bar{N}_{R}(r, f)+O(\log (r))(r \in I) .
$$


Corollary II.1.17.b: Let $f \in \mathcal{A}^{*}(D)$ and let $w_{1}, w_{2} \in \mathcal{A}^{0}(D)$ be distinct. Then

$$
T_{R}(r, f) \leq \bar{Z}_{R}\left(r, f-w_{1}\right)+\bar{Z}_{R}\left(r, f-w_{2}\right)+O(\log (r))(r \in I) .
$$

Proof. of Theorem II.1.17 Let $g=\frac{1}{f}, h_{j}=\frac{1}{w_{j}}, j=1,2, h_{3}=0$. Clearly,

$$
T_{R}(r, g)=T_{R}(r, f), T_{R}\left(r, h_{j}\right)=T_{R}\left(r, w_{j}\right), j=1,2,
$$

so we can apply Theorem II.13 to $g, h_{1}, h_{2}, h_{3}$. Thus we have:

$$
T_{R}(r, g) \leq \bar{Z}_{R}\left(r, g-h_{1}\right)+\bar{Z}_{R}\left(r, g-h_{2}\right)+\bar{Z}_{R}(r, g)+o\left(T_{R}(r, g)\right) .
$$

But we notice that $\bar{Z}_{R}\left(r, g-h_{j}\right)=\bar{Z}_{R}\left(r, f-w_{j}\right)$ for $j=1,2$ and $\bar{Z}_{R}(r, g)=$ $\bar{N}_{R}(r, f)$. Moreover, we know that $o\left(T_{R}(r, g)\right)=o\left(T_{R}(r, f)\right)$. Consequently, the claim is proven when $w_{1} w_{2}$ is not identically zero.

Now, suppose that $w_{1}=0$. Let $\lambda \in \mathbb{K}^{*}$, let $l=f+\lambda$ and $\tau_{j}=u_{j}+\lambda,(j=$ $1,2,3)$. Thus, we have $T_{R}(r, l)=T_{R}(r, f)+O(\log (r)), T_{R}\left(r, \tau_{j}\right)=T_{R}\left(r, w_{j}\right)+$ $O(\log (r)),(j=1,2), \bar{N}_{R}(r, l)=\bar{N}_{R}(r, f)$. By the claim already proven whenever $w_{1} w_{2} \neq 0$ we may write $\left.T_{R}(r, l) \leq \bar{Z}_{R}\left(r, l-\tau_{1}\right)+\bar{Z}_{R}\left(r, l-\tau_{2}\right)+\bar{N}_{R}(r, l)+o\left(T_{R}(r, l)\right)\right)$ hence $\left.T_{R}(r, f) \leq \bar{Z}_{R}\left(r, f-w_{1}\right)+\bar{Z}_{R}\left(r, f-w_{2}\right)+\bar{N}_{R}(r, l)+o\left(T_{R}(r, f)\right)\right)$.

\section{II.2. Applications of the Nevanlinna Theory out of a hole}

Theorems II.2.1 and II.2.2 are similar to Theorems I.8.2 and I.8.4:

Theorem II.2.1: $\quad$ Let $a_{1}, a_{2}, a_{3} \in \mathbb{K}\left(\right.$ with $\left.a_{i} \neq a_{j} \forall i \neq j\right)$ and let $f, g \in \mathcal{A}^{*}(D)$ satisfy $f^{-1}\left(\left\{a_{i}\right\}\right)=g^{-1}\left(\left\{a_{i}\right\}\right)(i=1,2,3)$. Then $f=g$.

Theorem II.2.2: Let $a_{1}, a_{2}, a_{3}, a_{4}, a_{5} \in \mathbb{K}$ (with $a_{i} \neq a_{j} \forall i \neq j$ ) and let $f, g \in \mathcal{M}^{*}(D)$ satisfy $f^{-1}\left(\left\{a_{i}\right\}\right)=g^{-1}\left(\left\{a_{i}\right\}\right)(i=1,2,3,4,5)$. Then $f=g$.

Proof. of Theorems II.2.1 and II.2.2: $\quad$ In both Theorems, we suppose that $f$ and $g$ are two different functions. Let us remark that since $f, g$ belong to $\mathcal{M}^{*}(D)$, by Theorem II.1.6 we have $\lim _{r \rightarrow+\infty} \frac{T_{R}(r, f)}{\log (r)}=+\infty$ and $\lim _{r \rightarrow+\infty} \frac{T_{R}(r, g)}{\log (r)}=+\infty$.

Now, for each $j=1, \ldots, n$, let $Y_{j}$ be the set of all zeros of $f-a_{j}$ (without taking multiplicities into account). Since $a_{i} \neq a_{j} \forall i \neq j$, we have $Y_{i} \cap Y_{j}=\emptyset \forall i \neq j$. Next, we notice that $f(x)=a_{j}$ implies $f(x)-g(x)=0$. Consequently, we check that

$$
\sum_{j=1}^{n} \bar{Z}_{R}\left(r, f-a_{j}\right) \leq \bar{Z}_{R}(r, f-g) .
$$

By applying Theorem II.1.15 to $f$ we obtain

$$
\begin{gathered}
(n-1) T_{R}(r, f) \leq \sum_{j=1}^{n} \bar{Z}_{R}\left(r, f-a_{j}\right)+\bar{N}_{R}(r, f)+O(\log (r)) \leq \\
\leq n \bar{Z}_{R}(r, f-g)+\bar{N}_{R}(r, f)+O(\log (r))(r \in I)
\end{gathered}
$$


hence by (1),

$$
(n-1) T_{R}(r, f) \leq T_{R}(r, f-g)+\bar{N}_{R}(r, f)+O(\log (r))(r \in I)
$$

and finally

$$
(n-1) T_{R}(r, f) \leq T_{R}(r, f-g)+N_{R}(r, f)+O(\log (r))(r \in I) .
$$

Similarly,

$$
(n-1) T_{R}(r, g) \leq T_{R}(r, f-g)+N_{R}(r, g)+O(\log (r))(r \in I),
$$

therefore we obtain

$$
\begin{gathered}
(n-1) \max \left(T_{R}(r, f), T_{R}(r, g)\right) \\
\left.\leq T_{R}(r, f-g)\right)+\max \left(N_{R}(r, f), N_{R}(r, g)\right)+O(\log (r))(r \in I) .
\end{gathered}
$$

Assume first we are in the hypothesis of Theorem II.2.1. We have $N_{R}(r, f)=$ $N_{R}(r, g)=0$, and by Theorem II.1.7, $T_{R}(r, f-g) \leq \max \left(T_{R}(r, f), T_{R}(r, g)\right)+$ $O(\log (r))$. Consequently,

$$
(n-1) \max \left(T_{R}(r, f), T_{R}(r, g)\right) \leq \max \left(T_{R}(r, f), T_{R}(r, g)\right)+O(\log (r))(r \in I) .
$$

Then by the remark at the beginning of the proof, we can see that the inequality does not hold with $n=3$, when $r$ goes to $+\infty$. Consequently, $f=g$.

Assume now the hypothesis of Theorem II.2.2. Since

$$
\max \left(N_{R}(r, f), N_{R}(r, g)\right) \leq \max \left(T_{R}(r, f), T_{R}(r, g)\right),
$$

by (2) and by Theorem II.1.7 we have

$$
(n-1) \max \left(T_{R}(r, f), T_{R}(r, g)\right) \leq 3 \max \left(T_{R}(r, f), T_{R}(r, g)\right)+O(\log (r))(r \in I) .
$$

The inequality does not hold with $n=5$, when $r$ goes to $+\infty$, hence $f=g$.

We can now apply the Second Main Theorem to obtain results concerning certain algebraic curves:

Theorem II.2.3: Let $\Lambda$ be a curve of equation $y^{q}=P(x)$ with $P \in \mathbb{K}[x]$. If $\Lambda$ admits a parametrization of the form $y=g(t), x=f(t)$ with $f, g \in \mathcal{M}(D)$ and if $f$ (resp. g) belongs to $\mathcal{M}^{0}(D)$, then $g$ (resp. $f$ ) also belongs to $\mathcal{M}^{0}(D)$.

Proof. Let $a_{1}, \ldots, a_{n}$ be $n$ distinct zeros of $P$. Suppose that two functions $f, g \in \mathcal{M}(D)$ satisfy the equation $g(t)^{q}=P(f(t))$. If $f$ belongs to $\mathcal{M}^{0}(D)$, then by Theorem II.1.6, $g$ also belongs to $\mathcal{M}^{0}(D)$. Conversely, if $g$ belongs to $\mathcal{M}^{0}(D)$, then $f$ satisfies $P(f)-g^{q}=0$, hence by Theorem II.1.6, $f$ belongs to $\mathcal{M}^{0}(D)$.

Theorem II.2.4: Let $\Lambda$ be a non-degenerate elliptic curve of equation $y^{2}=\left(x-a_{1}\right)\left(x-a_{2}\right)\left(x-a_{3}\right)$. There do not exist $g, f \in \mathcal{A}^{*}(D)$ such that $g(t)=$ $y, f(t)=x, t \in \mathbb{K}$. 
Proof. Indeed, suppose that there exist $g, f \in \mathcal{A}^{*}(D)$ such that $g(t)=$ $y, f(t)=x, t \in \mathbb{K}$. Since for each $j=1,2,3, f-a_{i}$ must have infinitely many zeros in $D$, each one is a zero of $g^{2}$ and hence it is a zero of even order, hence $\bar{Z}_{R}\left(r, f-a_{j}\right)=\frac{Z_{R}\left(r, f-a_{j}\right)}{2}$. Consequently, by Theorem II.1.15, we have

$$
2 T_{R}(r, f) \leq \sum_{j=1}^{3} \frac{Z_{R}\left(r, f-a_{j}\right)}{2}+O(\log (r)) \leq \frac{3 T_{R}(r, f)}{2}+O(\log (r)),
$$

a contradiction when $f \in \mathcal{A}^{*}(D)$.

Theorem II.2.5: Let $\Lambda$ be a curve of equation $y^{q}=P(x)$ with $P \in \mathbb{K}[x]$ admitting $n$ distinct zeros of order 1 . If $\Lambda$ admits a parametrization of the form $y=g(t), x=f(t)$ with $f, g \in \mathcal{M}^{*}(D)$ and $t \in D$, then $n \leq \frac{2 q}{q-1}$. Moreover, if $\operatorname{deg}(P)=n$ and if $n$ and $q$ are relatively prime, then $n \leq \frac{q+1}{q-1}$.

Proof. Let $a_{1}, \ldots, a_{n}$ be $n$ distinct zeros of $P$. Suppose that two functions $f, g \in \mathcal{M}^{*}(D)$ satisfy the equation $(g(t))^{q}=P(f(t))$. Let $\alpha \in D$ be a zero of $f-a_{j}$ of order $s$. It is a zero of order $l$ of $g-a_{j}$, hence $l q=s$ therefore $q$ divides $s$.

Consequently, for each $j=1, \ldots, n$, we have $\bar{Z}_{R}\left(r, f-a_{j}\right) \leq \frac{1}{q} Z_{R}\left(r, f-a_{j}\right)$, therefore, by Theorems II.1.7 and II.1.15 we have

$$
(n-1) T_{R}(r, f) \leq \frac{n}{q} T_{R}(r, f)+\bar{N}_{R}(r, f)+O(\log (r)) .
$$

Since $f, g$ belong to $\mathcal{M}^{*}(D)$, that implies $n \leq \frac{2 q}{q-1}$.

Suppose now that $\operatorname{deg}(P)=n$ and that $n$ and $q$ are relatively prime. Let $\beta$ be a pole of $f$ of order $l$ in $D$. Since $\operatorname{deg}(P)=n$, all zeros of $P$ are of order 1 and $\beta$ is a pole of $g^{q}$ of order $l n$. But since $n$ and $q$ are relatively prime, $q$ must divide $l$. Consequently, by (1) now we have

$$
(n-1) T_{R}(r, f) \leq \frac{n}{q} T_{R}(r, f)+\frac{1}{q} T_{R}(r, f)+O(\log (r))
$$

hence $(n-1) T_{R}(r, f) \leq\left(\frac{n+1}{q}\right) T_{R}(r, f)+O(\log (r))$. And since $f, g$ belong to $\mathcal{M}^{*}(D)$, that implies $n \leq \frac{q+1}{q-1}$.

By Theorems II.2.3 and II.2.5, we can get these corollaries:

Corollary II.2.5.a: Let $\Lambda$ be a curve of equation $y^{q}=P(x)$ with $q \geq 2, P \in \mathbb{K}[x]$ admitting $n$ distinct zeros of order 1 . If $\Lambda$ admits a parametrization of the form $y=g(t), x=f(t)$ with $f, g \in \mathcal{M}(D)$ and $t \in D$ and if $n>\frac{2 q}{q-1}$ then the two both functions $f$ and $g$ belong to $\mathcal{M}^{0}(D)$.

Corollary II.2.5.b: Let $\Lambda$ be a curve of equation $y^{q}=P(x)$ with $q \geq 2$ relatively prime to $n$ and $P \in \mathbb{K}[x]$ of degree $n$ admitting $n$ distinct zeros. If $\Lambda$ admits a parametrization of the form $y=g(t), x=f(t)$ with $f, g \in \mathcal{M}(D)$ and if $n>\frac{q+1}{q-1}$, then the two both functions $f$ and $g$ belong to $\mathcal{M}^{0}(D)$. 
Particularly Corollary II.2.5.b applies to hyper-elliptic curves.

Example: Let $\Lambda$ be a curve of equation $y^{2}=P(x)$ with $\operatorname{deg}(P)=5, P$ admitting five distinct zeros. If two functions $f, g \in \mathcal{M}(D)$ satisfy $g(t)^{2}=P(f(t))$, then the two both functions belong to $\mathcal{M}^{0}(D)$.

Another application concerns analytic functions:

Theorem II.2.6: Let $f, g \in \mathcal{M}(\mathbb{K})$ satisfy $g^{m}+f^{n}=1$, with $\min (m, n) \geq 3$ with $(m, n) \neq(3,3)$. Then $f$ and $g$ belong to $\mathcal{M}^{0}(D)$. Moreover, if $f, g \in \mathcal{A}(D)$ and satisfy $g^{m}+f^{n}=1$, with $\min (m, n) \geq 2$ and $(m, n) \neq(2,2)$, then $f$ and $g$ belong to, $\mathcal{A}^{0}(D)$.

Concerning the Hayman conjecture we can easily have a theorem that looks like this one for a disk, by following a similar way as in [24].:

Theorem II.2.7: Let $f \in \mathcal{M}(D)$. For every $n \geq 3, f^{\prime} f^{n}$ takes every value infinitely many times.

However, so far, here we don't have a theorem concerning $\mathcal{M}(D)$ in case $n=2$.

We can now consider the problem of branched functions.

Definition: Let $f \in \mathcal{M}^{*}(D)$ and let $w \in \mathcal{M}_{f}(D)$. Then $w$ is called a perfectly branched function, with respect to $f$ if all zeros of $f-w$ are multiple except maybe finitely many. Particularly, the definition applies to constants that are then called branched values.

Theorem II.2.8: Let $f \in \mathcal{M}^{*}(D)$. Then $f$ admits at most 4 perfectly branched values.

Proof. Suppose $f$ has $q$ perfectly branched values $b_{j}$ with $j=1, \ldots, q$. For each $j$, let $s_{j}$ be the number of simple zeros of $f-b_{j}$ and let $s=\sum_{j=1}^{q} s_{j}$. Applying Theorem II.1.15, we have

$$
(q-1) T_{R}(r, f) \leq \sum_{j=1}^{q} \bar{Z}_{R}\left(r, f-b_{j}\right)+\bar{N}_{R}(r, f)+O(\log r)
$$

But since $f-b_{j}$ has $s_{j}$ simple zeros, we have

$$
\begin{gathered}
\bar{Z}_{R}\left(r, f-b_{j}\right) \leq \frac{Z_{R}\left(r, f-b_{j}\right)+s_{j}(\log r-\log R)}{2}+O(1) \\
\leq \frac{T_{R}(r, f)+s_{j}(\log r-\log R)}{2}+O(1) \forall j=1, \ldots, q
\end{gathered}
$$

hence, by (1), we have

$$
(q-1) T_{R}(r, f) \leq \frac{q T_{R}(r, f)}{2}+T_{R}(r, f)+O(\log (r))
$$

By (2) clearly we have $q \leq 4$ in all cases, which shows the statement of Theorem II.2.8 whenever $f \in \mathcal{M}(D)$. 
In the proof of Theorem II.2.10, we will use the following lemma:

Lemma II.2.9. Let $\Theta(x)=\sum_{-\infty}^{0} a_{n} x^{n} \in \mathcal{A}(D)$, with $a_{0}=1$, have no zero in $D$. Take $R^{\prime}=4 R$ and set $D^{\prime}=\mathbb{K} \backslash d\left(0, R^{\prime-}\right)$. Then there exists a function $f \in \mathcal{A}\left(D^{\prime}\right)$ such that $(f(x))^{2}=\Theta(x)$.

Proof. Since $\Theta$ belongs to $\mathcal{A}(D)$ and has no zero in $D$, while $a_{0}=1$, we have $\left|a_{n}\right| R^{-n}<1 \forall n>0$ and $\lim _{n \rightarrow-\infty}\left|a_{n}\right| R^{-n}=0$. Then, there exists a unique function $\ell \in \mathcal{A}\left(d\left(1,\left(\frac{1}{4}\right)^{-}\right)\right)$with value in $d\left(1,1^{-}\right)$such that $(\ell(u))^{2}=u \quad \forall u \in d\left(1,\left(\frac{1}{4}\right)^{-}\right)$ (see for instance Theorem 31.23 in [12]). Here, we put $u=\sum_{-\infty}^{0} a_{n} x^{n}$. Since $\Theta$ has no zero in $D$, we have $\frac{\left|a_{n}\right|}{R^{n}}<1 \forall n>0$, hence $\frac{\left|a_{n}\right|}{(4 R)^{n}}<\frac{1}{4} \forall n>0$, therefore $\left.\Theta(x) \in d\left(1, \frac{1}{4}\right)^{-}\right)$. Consequently, we can apply $\ell$ to $\Theta(x)$ in $D^{\prime}$. Then, putting $f(x)=\ell(\Theta(x))$, we have $f(x)^{2}=\Theta(x) \forall x \in D^{\prime}$.

The following Theorem II.2.10 generalizes Theorem 50.12 in [12].

Theorem II.2.10: Let $f \in \mathcal{M}^{*}(D)$ have finitely many poles. Then $f$ admits at most one perfectly branched function in $\mathcal{M}^{0}(D)$.

ProOF. Suppose that $f$ admits two perfectly branched rational functions $w_{1}$ and $w_{2}$. If we consider the function $g=f-w_{1}$, we can see that $g$ has two perfectly branched rational functions 0 and $w_{1}-w_{2}$. So, without loss of generality, we may assume that $f$ admits two perfectly branched rational functions that are 0 and $w(x) \neq 0$.

Suppose first that $f$ has infinitely many zeros of order $\geq 3$. Then $Z_{R}(r, f)-$ $2 \bar{Z}_{R}(r, f)$ is a function $\zeta(r)$ such that

$$
\lim _{r \rightarrow+\infty} \frac{\zeta(r)}{\log r}=+\infty
$$

therefore

$$
\bar{Z}_{R}(r, f) \leq \frac{T_{R}(r, f)-\zeta(r)}{2} .
$$

On the other hand, by Theorem II.1.17, we have

$$
T_{R}(r, f) \leq \bar{Z}_{R}(r, f)+\bar{Z}_{R}(r, f-w)+\bar{N}_{R}(r, f)+o\left(T_{R}(r, f)\right) .
$$

Consequently by (1), we can see a contradiction proving that $f$ cannot admit 0 and $w$ as branched rational functions.

Suppose now that all zeros of both $f$ and $f-w$ are of order 2 except finitely many. Since $f$ has infinitely many zeros in $D$ and since $w \in \mathcal{M}^{0}(D)$, there exists $R^{\prime}>4 R$ satisfying the following properties:

i) all poles of $f$ in $D$ lie in $\Delta\left(0, R, R^{\prime}\right)$

ii) $|f|(r)>|w|(r) \forall r \geq R^{\prime}$

iii) all zeros of $f$ and of $f-w$ in $D \backslash \Delta\left(0, R, R^{\prime}\right)$ are of order 2 exactly.

Let $S^{\prime}=d\left(0, R^{\prime-}\right)$ and let $D^{\prime}=\mathbb{K} \backslash S^{\prime}$. Then $f$ obviously belongs to $\mathcal{M}\left(D^{\prime}\right)$. Therefore, by Theorem II.1.1, $f$ admits a Motzkin factor of the form $x^{s} \theta$ with $s=m\left(f, S^{\prime}\right)$ and then we can write $f$ in the form $x^{s} \theta g^{2}$ and $g \in \mathcal{A}(\mathbb{K})$, having 
no zero in $S^{\prime}$. Similarly, $f-w$ admits a Motzkin factor of the form $x^{t} \tau$ with $t=m\left(f-w, S^{\prime}\right)$ and we can then write $f-w$ in the form $x^{t} \tau h^{2}$ and $h \in \mathcal{A}(\mathbb{K})$, having no zero in $S^{\prime}$.

Since $|f|(r)>|w|(r) \forall r \geq R^{\prime}$, we can check that $f$ and $f-w$ have the same number of zeros on each circle $C(0, r)\left(r \geq R^{\prime}\right)$ and $|f|(r)=|f-w|(r) \forall r \geq R^{\prime}$. Consequently, $s=t$. We have the equality

$$
g^{2}-\frac{\tau}{\theta} h^{2}=\frac{b}{x^{s} \theta}
$$

Now, $\frac{\tau}{\theta}$ is a Motzkin factor of index zero, hence by Lemma II.2.9, it admits a square root $\Xi \in H\left(D^{\prime}\right)$ of the form $\sum_{-\infty}^{0} a_{n} x^{n}$ with $\left|a_{n}\right| S^{n}<\left|a_{0}\right| \forall n<0$. Then by (2) we have

$$
(g-\Xi h)(g+\Xi h)=\frac{w}{x^{s} \theta} .
$$

We will check that this equality is impossible. Indeed, both functions $g-\Xi h$ and $g+\Xi h$ belong to $\mathcal{A}\left(D^{\prime}\right)$. Suppose $Z_{R^{\prime}}(r, g-\Xi h)=O(\log (r))$. Then $g+\Xi h=$ $(g-\Xi h)+2 \Xi h$ satisfies $Z_{R^{\prime}}(r, g-\Xi h)+Z_{S}(r, \Xi h)=Z_{R^{\prime}}(r, \Xi h)+O(\log (r))$ and consequently,

because

$$
\lim _{r \rightarrow+\infty}\left[\frac{Z_{R^{\prime}}(r,(g-\Xi h)(g+\Xi h))}{\log (r)}\right]=+\infty
$$

$$
\lim _{r \rightarrow+\infty}\left[\frac{Z_{R^{\prime}}(r, \Xi h)}{\log (r)}\right]=+\infty .
$$

But on the other hand, by construction, $Z_{R^{\prime}}\left(r, \frac{w}{x^{s} \theta}\right)$ is of the form $O(\log (r))$, which shows that (3) is impossible. That ends the proof of Theorem II.2.10.

Corollary II.2.10.a: Let $f \in \mathcal{A}^{*}(D)$. Then $f$ admits at most one perfectly branched function in $\mathcal{M}^{0}(D)$.

Notation Let $h \in \mathcal{M}(\mathbb{K}) \backslash \mathbb{K}$ (resp. $h \in E(x) \backslash E$ ) and let $\Lambda(h)$ be the set of zeros $c$ of $h^{\prime}$ such that $h(c) \neq h(d)$ for every zero $d$ of $h^{\prime}$ other than $c$. If $\Lambda(h)$ is finite, we denote by $\Upsilon(h)$ its cardinal and if $\Lambda(h)$ is not finite, we put $\Upsilon(h)=+\infty$.

Given a property $\mathcal{Q}$ satisfied by $h$, we will denote by $Z_{R}\left(r, h^{\prime} \mid \mathcal{Q}\right)$ the counting function of zeros of $h^{\prime}$ whenever $\mathcal{Q}$ is satisfied.

In the proof of Theorems II.2.13 and II.2.14 we will need the following lemma which is similar to Lemma 10 in [11] (see also Lemma 53.1 in [12]):

Lemma II.2.11: Let $P(x) \in \mathbb{K}[x] \backslash \mathbb{K}$ and let $f, g \in \mathcal{M}(D)$ satisfy $P \circ f=P \circ g$. Let $W=\left\{c_{1}, \ldots, c_{l}\right\}$ be the set of zeros of $P^{\prime}$.

For each $j=1, \ldots, k(k \leq l)$ let $q_{j}=\omega_{c_{j}}\left(P^{\prime}\right)$. We assume that $P\left(c_{j}\right) \neq$ $P\left(c_{n}\right) \forall j=1, \ldots, k, \forall n \leq l$. Then $f, g$ satisfy

$$
\bar{N}_{R}(r, f)+\sum_{j=1}^{k} \bar{Z}_{R}\left(r, f-c_{j}\right) \leq \bar{Z}_{R}\left(r, \frac{1}{f}-\frac{1}{g}\right)+\sum_{j=1}^{k} \frac{1}{q_{j}} Z_{R}\left(r, g^{\prime} \mid f(x)=c_{j}, g(x) \notin W\right) .
$$

Furthermore, if $f, g \in \mathcal{A}(D)$, then 


$$
\sum_{j=1}^{k} \bar{Z}_{R}\left(r, f-c_{j}\right) \leq \bar{Z}_{R}(r, f-g)+\sum_{j=1}^{k} \frac{1}{q_{j}} Z_{R}\left(r, g^{\prime} \mid f(x)=c_{j}, g(x) \notin W\right) .
$$

Proof. Without loss of generality, we may assume that $0 \notin S$ and that $c_{n} \neq 0 \forall n \leq j$. Let $\mathcal{P}(f)$ be the set of poles of $f$ and let $\phi=\frac{1}{f}-\frac{1}{g}$. Since $P \circ f=P \circ g$, each pole $\alpha$ of $f$ is a pole of same order of $g$ and hence, we have $\phi(\alpha)=0$. Therefore

$$
\bar{N}_{R}(r, f) \leq \bar{Z}_{R}(r, \phi \mid x \in \mathcal{P}(f)) .
$$

Let us fix $j \in\{1, \ldots, k\}$ and let $\alpha \in D$ satisfy $f(\alpha)=c_{j}$. Suppose first that $g(\alpha)$ lies in $W$. Thanks to the hypothesis $P\left(c_{n}\right) \neq P\left(c_{j}\right) \forall n \neq j$, if $g(\alpha) \neq c_{j}$ then $P(g(\alpha)) \neq P\left(c_{j}\right)$, a contradiction to $P(g(\alpha))=P(f(\alpha))$. So we have $g(\alpha)=$ $f(\alpha)=c_{j}$ and since $c_{j} \neq 0$, then $\phi(\alpha)=0$. Consequently,

$$
\bar{Z}_{R}\left(r, f-c_{j} \mid g(x) \in W\right) \leq \bar{Z}_{R}\left(r, \phi \mid f(x)=c_{j}\right)
$$

and similarly if $f, g \in \mathcal{A}(D)$

$$
\bar{Z}_{R}\left(r, f-c_{j} \mid g(x) \in W\right) \leq \bar{Z}_{R}\left(r, f-g \mid f(x)=c_{j}\right) .
$$

Consequently, by (1) and (2) we can derive

$$
\bar{N}_{R}(r, f)+\sum_{j=1}^{k} \bar{Z}_{R}\left(r, f-c_{j} \mid g(x) \in W\right) \leq \bar{Z}_{R}(r, \phi) .
$$

Similarly, if $f, g \in \mathcal{A}(D)$, by (3) we have

$$
\sum_{j=1}^{k} \bar{Z}_{R}\left(r, f-c_{j} \mid g(x) \in W\right) \leq \bar{Z}_{R}(r, f-g) .
$$

In order to complete the proof, we will show

$$
\bar{Z}_{R}\left(r, f-c_{j} \mid g(x) \notin W\right) \leq \frac{1}{q_{j}} Z_{R}\left(r, g^{\prime} \mid f(x)=c_{j}, g(x) \notin W\right) .
$$

Indeed, consider $\alpha$ such that $g(\alpha) \notin W$

Since $P^{\prime}(f(\alpha))=P^{\prime}\left(c_{j}\right)=0$, we notice that $f^{\prime}(\alpha) P^{\prime}(f(\alpha))=g^{\prime}(\alpha) P^{\prime}(g(\alpha))=$ 0 . But since $g(\alpha) \notin W$, we have $P^{\prime}(g(\alpha)) \neq 0$, hence $g^{\prime}(\alpha)=0$. Consequently, we obtain

$$
\bar{Z}_{R}\left(r, f-c_{j} \mid g(x) \notin W\right) \leq \bar{Z}_{R}\left(r, g^{\prime} \mid f(x)=c_{j}, g(x) \notin W\right) .
$$

On the other hand, since $f(\alpha)=c_{j}$, we see that $\omega_{\alpha}\left(f^{\prime}(x) P^{\prime}(f(x))\right) \geq q_{j}$ hence

$$
\omega_{\alpha}\left(g^{\prime}(x) P^{\prime}(g(x))\right) \geq q_{j} .
$$

But since $g(\alpha) \notin W$, we have $P^{\prime}(g(\alpha)) \neq 0$, hence by $(8), \omega_{\alpha}\left(g^{\prime}\right) \geq q_{j}$, and consequently

(9) $\quad \bar{Z}_{R}\left(r, g^{\prime} \mid f(x)=c_{j}, g(x) \notin W\right) \leq \frac{1}{q_{j}} Z_{R}\left(r, g^{\prime} \mid f(x)=c_{j}, g(x) \notin W\right)$. 
Thus, by (24) and (26) we obtain (23) which, by (4) proves

$\bar{N}_{R}(r, f)+\sum_{j=1}^{k} \bar{Z}_{R}\left(r, f-c_{j}\right) \leq \bar{Z}_{R}\left(r, \frac{1}{f}-\frac{1}{g}\right)+\sum_{j=1}^{k} \frac{1}{q_{j}} Z_{R}\left(r, g^{\prime} \mid f(x)=c_{j}, g(x) \notin W\right)$.

Similarly, if $f, g \in \mathcal{A}(D)$, by (5) and (6) we have

$$
\sum_{j=1}^{k} \bar{Z}_{R}\left(r, f-c_{j}\right) \leq \bar{Z}_{R}(r, f-g)+\sum_{j=1}^{k} \frac{1}{q_{j}} Z_{R}\left(r, g^{\prime} \mid f(x)=c_{j}, g(x) \notin W\right) .
$$

Lemma II.2.12: Let $P \in \mathbb{K}[x]$ and let $f, g \in \mathcal{A}^{*}(D)$ satisfy $P \circ f=P \circ g$. Then $\left.T_{R}(r, f)=T_{R}(r, g)+O(\log (r)) r>R\right)$.

Proof. Let $q=\operatorname{deg}(P)$. By Theorem T.II.5, we have $T_{R}(r, P \circ f)=q T_{R}(r, f)+$ $O(\log (r))$ and $T_{R}(r, P \circ g)=q T_{R}(r, g)+O(\log (r))$. But since, $P \circ f=P \circ g$ that means $T_{R}(r, f)=T_{R}(r, g)+O(\log (r))$.

Theorem II.2.13: Let $P \in \mathbb{K}[x]$ be such that $\Upsilon(P) \geq 2$. Then $P$ is a polynomial of uniqueness for $\mathcal{A}^{*}(D)$.

Remark: One can show that when a polynomial is of degree 3 and such that $P^{\prime}$ has 2 distinct zeros, then the condition $\Upsilon(P)=2$ is automatically satisfied.

Example: Without loss of generality we can assume that $P^{\prime}$ is of the form $3 x^{2}-b$, hence $P(x)=x^{3}-b x+c$. Then $P(b)=b^{3}-b^{2}+c, P(-b)=-b^{3}+b^{2}+c \neq P(b)$.

Theorem II.2.14: Let $P \in \mathbb{K}[x]$ be such that $\Upsilon(P) \geq 4$. Then $P$ is a polynomial of uniqueness for $\mathcal{M}^{*}(D)$.

Example: 1) Let $P(x)=\frac{x^{4}}{4}+\frac{x^{3}}{3}-x^{2}$. Then $P^{\prime}(x)=x(x-1)(x+2)$.

And $P(0)=0, P(1)=\frac{1}{4}+\frac{1}{3}-1, P(2)=\frac{8}{3}$.

Thus the three zeros $a_{j}$ of $P^{\prime}$ satisfy $P\left(a_{i}\right) \neq P\left(a_{j}\right) \forall i \neq j$. Consequently, $\Upsilon(P)=3$ and hence $P$ is a polynomial of uniqueness for $\mathcal{A}^{*}(D)$.

2) Let $P(x)=\frac{x^{5}}{5}-\frac{5 x^{3}}{3}+4 x$. Then

$P^{\prime}(x)=(x-1)(x+1)(x-2)(x+2) . P(1)=\frac{1}{5}-\frac{5}{3}+4$,

$P(-1)=-P(-1), P(2)=\frac{32}{5}-\frac{24}{3}+8=-P(-2)$.

Thus the four zeros $a_{j}$ of $P^{\prime}$ satisfy $P\left(a_{i}\right) \neq P\left(a_{j}\right) \forall i \neq j$. Consequently, $\Upsilon(P)=4$ and hence $P$ is a polynomial of uniqueness for $\mathcal{M}^{*}(D)$. 
Proof. of Theorems II.2.13 and II.2.14 Suppose that $f$ and $g$ are not identical. Let $W$ be the set of zeros of $P^{\prime}$ and let $c_{1}, \ldots, c_{k}$ lie in $W$. Clearly by applying Theorem II.1.15 we obtain respectively in Theorems II.2.13 and II.2.14

(1) $(k-1) T_{R}(r, f) \leq \sum_{j=1}^{k} \bar{Z}_{R}\left(r, f-c_{j}\right)+\bar{N}_{R}(r, f)-Z_{R}^{W}\left(r, f^{\prime}\right)+O(\log (r)),(r>R)$,

(2) $(k-1) T_{R}(r, g) \leq \sum_{j=1}^{k} \bar{Z}_{R}\left(r, g-c_{j}\right)+\bar{N}_{R}(r, g)-Z_{R}^{W}\left(r, g^{\prime}\right)+O(\log (r)),(r>R)$.

Now, let $\phi=\frac{1}{f}-\frac{1}{g}$ and for each $j=1, \ldots, k$, let $q_{j}=\omega_{c_{j}}\left(P^{\prime}\right)$. By $(1)$ and $(2)$ and by Lemma II.2.11 we obtain

$$
(k-1) T_{R}(r, f) \leq \bar{Z}_{R}(r, \phi)+\sum_{j=1}^{k} \frac{1}{q_{j}} Z_{R}\left(r, g^{\prime} \mid f(x)=c_{j}, g(x) \notin W\right)
$$

$$
-Z_{R}\left(r, f^{\prime} \mid f(x) \notin W+O(\log (r))\right.
$$

and similarly:

$$
\begin{aligned}
(k-1) T_{R}(r, g) \leq & \bar{Z}_{R}(r, \phi)+\sum_{j=1}^{k} \frac{1}{q_{j}} Z_{R}\left(r, f^{\prime} \mid g(x)=c_{j}, f(x) \notin W\right) \\
& -Z_{R}\left(r, g^{\prime} \mid g(x) \notin W+O(\log (r)) .\right.
\end{aligned}
$$

By adding in each case the two inequalities by (3) and (4) in Theorems II.2.13 and II.2.14, we obtain:

$$
\begin{gathered}
(k-1)\left(T_{R}(r, f)+T_{R}(r, g)\right) \leq 2 \bar{Z}_{R}(r, \phi)+ \\
+\sum_{j=1}^{k} \frac{1}{q_{j}}\left[Z_{R}\left(r, f^{\prime} \mid g(x)=c_{j}, f(x) \notin S+Z_{R}\left(r, g^{\prime} \mid f(x)=c_{j}, g(x) \notin W\right)\right]\right. \\
-Z_{R}\left(r, f^{\prime} \mid f(x) \notin W\right)-Z_{R}\left(r, g^{\prime} \mid g(x) \notin W\right)+O(\log (r)) .
\end{gathered}
$$

Now, in each inequality (5), we notice that in the left side member we have the term:

$$
\sum_{j=1}^{k} \frac{1}{q_{j}}\left[Z_{R}\left(r, f^{\prime} \mid g(x)=c_{j}, f(x) \notin S\right)\right]-Z_{R}\left(r, f^{\prime} \mid f(x) \notin W\right)
$$

which is clearly less than or equal to zero and similarly

$$
\sum_{j=1}^{k} \frac{1}{q_{j}}\left[Z_{R}\left(r, g^{\prime} \mid f(x)=c_{j}, g(x) \notin S\right)\right]-Z_{R}\left(r, g^{\prime} \mid g(x) \notin W\right) \leq 0 .
$$


Consequently, by Lemma II.2.11, in Theorems II.2.14 we obtain

$$
(k-1)\left(T_{R}(r, f)+T_{R}(r, g)\right) \leq 2 \bar{Z}_{R}(r, \phi)+O(\log (r))
$$

and hence

$$
(k-1)\left(T_{R}(r, f)+T_{R}(r, g)\right) \leq 2 \bar{Z}_{R}(r, f-g)+O(\log (r)) .
$$

Now, by Theorem II.1.7 we have $Z_{R}(r, \phi) \leq T_{R}(r, f)+T_{R}(r, g)+O(\log (r))$ therefore $k \leq 3$ and hence, if $\Upsilon(P) \geq 4$, we have $f=g$.

Now assume the hypotheses of Theorem II.2.13. By Lemma II.2.11 we can replace $Z_{R}(r, \phi)$ by $Z_{R}(r, f-g)$. Next, by Lemma II.2.12, $T_{R}(r, f)=T_{R}(r, g)+$ $O(\log (r))$, hence, by Theorem II.1.7, we can derive $T_{R}(r, f-g) \leq T_{R}(r, f)+$ $O(\log (r))=T_{R}(r, g)+O(\log (r))$. Consequently in place of $(6)$, in Theorem II.2.13 we obtain

$(k-1)\left(T_{R}(r, f)+T_{R}(r, g)\right) \leq 2 \bar{Z}_{R}(r, f-g)+O(\log (r)) \leq T_{R}(r, f)+T_{R}(r, g)+O(\log (r))$.

Thus we can conclude that $k \leq 2$ in Theorem II.2.13 and hence, if $\Upsilon(P) \geq 3$, we have $f=g$.

Remark: The hypothesis $\Upsilon(P) \geq 4$ however is not necessary to prove that a polynomial $P$ is a polynomial of uniqueness for $\mathcal{M}^{*}(D)$, as shows Theorem II.2.16 below. The proof is similar to that of Theorem 41.9 in [12] and first came from [14]:

In the proof of Theorem II.2.16 we will need the following basic Lemma II.2.15 (stated in [12]).

Lemma II.2.15: $\quad$ Let $E$ be an algebraically closed field of characteristic 0 and let $P(x)=(n-1)^{2}\left(x^{n}-1\right)-n(n-2)\left(x^{n-1}-1\right)^{2} \in E[x]$. Then $P$ admits 1 as a zero of order 4 and all other zeros $u_{j}(1 \leq j \leq 2 n-6)$ are simple.

Theorem II.2.16: Let

$$
Q(x)=\left((n+2)(n+1) x^{n+3}-2(n+3)(n+1) x^{n+2}+(n+3)(n+2) x^{n+1}\right)
$$

with $b \in \mathbb{K}^{*}$. Then $Q$ is a polynomial of uniqueness for $\mathcal{M}^{*}(D)$ for every $n \geq 3$.

Proof. Suppose $f, g \in \mathcal{M}(D)$ and suppose that $Q(f)=Q(g)$. Let $h=\frac{f}{g}$. We can derive

$(n+2)(n+1)\left(h^{n+3}-1\right) g^{2}-2(n+3)(n+1)\left(h^{n+2}-1\right) g+(n+3)(n+2)\left(h^{n+1}-1\right)=0$.

If $h$ is a constant, it is 1 , a contradiction. So, we suppose $h$ is not constant.

Let $\left.P(x)=(n+2)^{2}\left(x^{n+3}\right)-1\right)-(n+3)(n+1)\left(x^{n+2}-1\right)^{2} \in \mathbb{K}[x]$. By Lemma II.2.15 $P$ admits 1 as a zero of order 4 and all other zeros $u_{j}(1 \leq j \leq 2 n)$ are simple. By change of variable, we can obviously assume that $h-u_{j}$ has no zero and no pole at 0 . Consequently, we check that

$$
\left(g-\left(\frac{n+3}{n+2}\right)\left(\frac{h^{n+2}-1}{h^{n+3}-1}\right)\right)^{2}=\frac{(n+3)(h-1)^{4} \prod_{j=1}^{2 n}\left(h-u_{j}\right)}{(n+2)^{2}(n+1)\left(h^{n+3}-1\right)^{2}} .
$$


Since $\frac{(n+3)(h-1)^{4} \prod_{j=1}^{2 n}\left(h-u_{j}\right)}{(n+2)^{2}(n-+1)\left(h^{n+3}-1\right)^{2}}$ is equal to a square, clearly each zero of $h-$ $u_{j},(1 \leq j \leq 2 n)$ has order at least 2 . Consequently

$$
\sum_{j=1}^{2 n} \bar{Z}_{R}\left(r, h-u_{j}\right) \leq \frac{1}{2} \sum_{j=1}^{2 n} Z_{R}\left(r, h-u_{j}\right) \leq \frac{1}{2}(2 n) T_{R}(r, h)+O(1)(r>R) .
$$

Then, applying Theorem II.1.15 to $h$ at the points $u_{j}(1 \leq j \leq 2 n)$, we obtain

$$
\begin{gathered}
(2 n-1) T_{R}(r, h) \leq \sum_{j=1}^{2 n} \bar{Z}_{R}\left(r, h-u_{j}\right)+\bar{N}_{R}(r, h)+O(\log (r)) \leq \\
\leq \frac{1}{2} \sum_{j=1}^{2 n} Z_{R}\left(r, h-u_{j}\right)+\bar{N}_{R}(r, h)+O(\log (r)) \\
\leq \frac{1}{2}(2 n) T_{R}(r, h)+\bar{N}_{R}(r, h)+O(\log (r))(r>R)
\end{gathered}
$$

and therefore $(2 n-1) T_{R}(r, h) \leq n T_{R}(r, h)+T_{R}(r, h)+O(\log (r))(r>R)$. Now, since $f, g$ belong to $\mathcal{M}^{*}(D)$, by Theorem II.1.6 we have $\lim _{r \rightarrow+\infty} \frac{T_{R}(r, h)}{\log (r)}=+\infty$ and hence we can conclude that $n \leq 2$. That finishes the proof of Theorem II.2.16.

Notation: Following Theorem II.2.16, given $n \in \mathbb{N}$ and let $c \in \mathbb{K}$ ) we denote by $P_{n, c}$ the polynomial introduced in [12] and also used in [11]:

$P_{n, c}(x)=(n-1)(n-2) x^{n}-2 n(n-2) x^{n-1}+n(n-1) x^{n-2}+c$ and by $L(n, c)$ be the set of zeros of $P_{n, c}$ in $\mathbb{K}$.

In the proof of Theorems II.2.19 and II.2.20, we will also need the following lemmas given in [5]: and [14]:

Lemma II.2.17: Let $F, G \in \mathcal{M}(D)$ have the same poles, ignoring multiplicitiy, and let $H=\frac{F^{\prime \prime}}{F^{\prime}}-\frac{G^{\prime \prime}}{G^{\prime}}$. Every pole of $H$ has multiplicity order 1 . Let $\alpha$ be a pole of $F$ and $G$. If $\alpha$ has same multiplicity for $F$ and $G$, then $H$ has no pole at $\alpha$. Moreover, if $\alpha$ has a multiplicity order 1 for both $F$ and $G$, then $\alpha$ is a zero of $H$.

Lemma II.2.18: Let $f, g \in \mathcal{M}(D))$ be two different non-constant functions satisfying $\frac{f^{\prime \prime}}{f^{\prime}}=\frac{g^{\prime \prime}}{g^{\prime}}$. Then $f$ and $g$ are linked by a relation of the form $f=a g+b$.

Theorem II.2.19: Let $f, g \in \mathcal{M}^{*}(D)$ be two different non-constant functions satisfying $f^{-1}(L(n, c))=g^{-1}(L(n, c))$. Then $n \leq 16$. Moreover, if $f, g \in \mathcal{A}^{*}(D)$, then $n \leq 9$.

Corollary II.2.19.a: Let $n \geq 17$. Then $L(n, c)$ is an ursim for $\mathcal{M}^{*}(D)$. Let $n \geq 10$. Then $L(n, c)$ is an ursim for $\mathcal{A}^{*}(D)$.

In order to state Theorem II.2.20, we need to recall the notation $\mathcal{E}$ used with URSCM. Given a subset $B$ of $\mathbb{K}$ and $f \in \mathcal{M}(D)$ we denote by $\mathcal{E}(f, B)$ the set in $\mathbb{K} \times \mathbb{N}^{*}$ : 
$\bigcup_{a \in B}\left\{(z, q) \in \mathbb{K} \times \mathbb{N}^{*} \mid z\right.$ a zero of order $q$ of $\left.f(x)-a\right\}$.

And given a subset $B$ the projective space of dimension 1 on $\mathbb{K}, \mathbb{P}^{1}(\mathbb{K})$, containing $\{\infty\}$ and $f \in \mathcal{M}(D)$, we denote by $\mathcal{E}(f, B)$ the subset of $\mathbb{K} \times \mathbb{N}^{*}$ : $\mathcal{E}(f, S \cap \mathbb{K}) \cup\{(z, q) z$ a pole of order $q$ of $f\}$.

Theorem II.2.20: Let $f, g \in \mathcal{M}^{*}(D)$ be two distinct non-constant functions satisfying $\mathcal{E}(f, L(n, c))=\mathcal{E}(g, L(n, c))$. Then $n \leq 10$. Moreover, if $f, g \in \mathcal{A}^{*}(D)$ then $n \leq 6$.

Corollary II.2.20.a: For every $n \geq 11, L(n, c)$ is an urscm for $\mathcal{M}^{*}(D)$ and for every $n \geq 7, L(n, c)$ is an urscm for $\mathcal{A}^{*}(D)$.

Proof. of Theorems II.2.19 and II.2.20: In order to simplify the notation, we will just denote by $P$ the polynomial $P_{n, c}$ and by $L$ the set $L(n, c)$.

We then consider two distinct non-constant meromorphic functions $f, g \in$ $\mathcal{M}(D)$ and we assume $f^{-1}(L)=g^{-1}(L)$ in Theorem II.2.19 and $\mathcal{E}(f, S)=\mathcal{E}(g, S)$ in Theorem II.2.20.

Let $F=\frac{1}{P(f)} \quad G=\frac{1}{P(g)}$ and $H=\frac{F^{\prime \prime}}{F^{\prime}}-\frac{G^{\prime \prime}}{G^{\prime}}$.

We first suppose that $H$ is identically 0 . By Lemma II.2.18, it is immediate to derive that there exist $A \in \mathbb{K}^{*}$ and $B \in \mathbb{K}$ such that $P(f)=\frac{P(g)}{B P(g)+A}$. Therefore, by Theorems II.1.7 we have: $(1) \quad T_{R}(r, f)=T_{R}(r, g)+O(\log (r))(r \in$ ] $0, R[)$.

We can check that $P(X)+c$ is of the form $b X^{n-2}\left(X-e_{1}\right)\left(X-e_{2}\right)+c$, with $b, e_{1}, e_{2} \in \mathbb{K}$, (resp. $\left.b, e_{1}, e_{2} \in \mathbb{K}\right)$ and $e_{1} e_{2} \neq 0, e_{1} \neq e_{2}$.

We have to distinct three cases: $i) B \neq 0$, ii) $B=0, A=1$, iii) $B=0, A \neq 1$.

i) Since $A B \neq 0$, every zero of $P(g)+\frac{A}{B}$ is a pole of $P(f)$ and therefore is a zero of order at least $n$ of $P(g)+\frac{A}{B}$. On the other hand, we check that whenever $A, B \in \mathbb{K}^{*}$, the polynomial $P(X)+\frac{A}{B}$ admits at least two distinct zeros $b_{1}$ and $b_{2}$ of order 1 and therefore it admits another zero $l$ of order at most $n-2$. Consequently, every zero of $g-b_{1}$ or $g-b_{2}$ has order at least $n$ and every zero of $g-l$ has order at least 2 . By a change of variable, we can obviously assume that $g, g-l, g-b_{1}, g-b_{2}$ have neither any zeros nor any poles at 0 . Then, by applying Theorem II.1.15, we obtain:

$$
\begin{aligned}
& 2 T_{R}(r, g)-\bar{N}_{R}(r, g) \leq \bar{Z}_{R}(r, g-l)+\bar{Z}_{R}\left(r, g-b_{1}\right)+\bar{Z}_{R}\left(r, g-b_{2}\right)+O(\log (r)) \leq \\
& \quad \leq \frac{1}{2} Z_{R}(r, g-l)+\frac{1}{n}\left(Z_{R}\left(r, g-b_{1}\right)+Z_{R}\left(r, g-b_{2}\right)\right)+O(\log (r))(r \in J)
\end{aligned}
$$

which leads to $n \leq 4$. ii) In this case we have $P(f)=P(g)$. But by Theorem

II.2.15, $P$ is a polynomial of uniqueness, for $\mathcal{M}_{u}\left(d\left(0, R^{-}\right)\right)$, hence $f=g$.

iii) Let $\lambda=\frac{1}{A}$. We will check that at least one of the two polynomials $Q_{1}(X)=(n-1)(n-2) X^{n}-2 n(n-2) X^{n-1}+n(n-1) X^{n-2}+c(\lambda-1)$ and 
$Q_{2}(X)=(n-1)(n-2) X^{n}-2 n(n-2) X^{n-1}+n(n-1) X^{n-2}+c\left(\frac{1}{\lambda}-1\right)$ admits $n$ distinct zeros. Indeed, suppose this not true. We notice that $Q_{1}^{\prime}(X)=Q_{2}^{\prime}(X)=P^{\prime}(X)=n(n-1)(n-2) X^{n-3}(X-1)^{2}$. Consequently, if both $Q_{1}$ and $Q_{2}$ admit at least one zero of order $>1$, then this must be 1 , because it cannot be 0 . But then, we have $c(\lambda-1)=c\left(\frac{1}{\lambda}-1\right)=-2$. Since $c \neq 0$, and since $\lambda \neq 1$, this implies $\lambda=-1$, and $c=1$, which is excluded by hypothesis.

Now, since $\lambda$ and $\frac{1}{\lambda}$ play symmetric roles, without loss of generality we can assume that $Q_{1}$ admits $n$ distinct zeros $b_{1}, \ldots, b_{n}$. Thus, putting $\gamma(X)=\lambda X^{n-2}(n-1)\left((n-2) X^{2}-2 n(n-2) X+n(n-1)\right)$, we have (2) $Q_{1}(f)=\gamma(g)$.

So, applying Theorem II.1.15, we obtain

$$
\left.(n-1) T_{R}(r, f) \leq \sum_{j=1}^{n} \bar{Z}_{R}\left(r, f-b_{j}\right)+\bar{N}_{R} r, f\right)+O(\log (r))(r \in J) .
$$

On the other hand, we have

$$
\begin{gathered}
\sum_{j=1}^{n} \bar{Z}_{R}\left(r, f-b_{j}\right) \leq \bar{Z}_{R}\left(r, Q_{1}(f)\right)=\bar{Z}_{R}(r, g)+\bar{Z}_{R}\left(r, g-e_{1}\right)+\bar{Z}_{R}\left(r, g-e_{2}\right) \\
\leq 3 T_{R}(r, g)+O(\log (r)) .
\end{gathered}
$$

But by (2) it is seen that $T_{R}(r, g) \leq T_{R}(r, f)+O(\log (r))(r \in J)$, hence finally

$$
(n-1) T_{R}(r, f) \leq 3 T_{R}(r, f)+\bar{N}_{R}(r, f)+O(\log (r))(r \in J) .
$$

Consequently, we have $n \leq 5$. Moreover, if $f, g \in \mathcal{A}\left(d\left(0, R^{-}\right)\right)$, then $N_{R}(r, f)=0$, and then (3) leads to $n \leq 4$. That finishes proving the claims of the Theorems when $H=0$.

Henceforth, we suppose that $H$ is not identically 0 and we will apply Fujimoto's method [15]. Let $\alpha$ be a zero of $f-a_{j}$, for some $j$. Then both $P(f)$ and $P(g)$ vanish at $\alpha$. So, we have $\sum_{j=1}^{n} \bar{Z}_{R}\left(r, f-a_{j}\right)=\bar{Z}_{R}(r, P(f))=\bar{Z}_{R}(r, P(g))$. According to

Lemma II.2.17, if both $P(f), P(g)$ have a zero of order one, then $H$ also has a zero. Else, at least one of the two functions $P(f)$ and $P(g)$ admits $\alpha$ as a zero of order strictly greater than 1 , and then, (since all zeros of $P$ are simple), at least one of the two functions $f^{\prime}, g^{\prime}$ has a zero at $\alpha$, while $\alpha$ is a zero $f-a_{i}$ for some $i$ and a zero of $g-a_{j}$ for some $j$. Consequently, we obtain

$$
\sum_{j=1}^{n} \bar{Z}_{R}\left(r, f-a_{j}\right) \leq \bar{Z}_{R}(r, H)+\bar{Z}_{R}\left(r, f^{\prime}\right)+\bar{Z}_{R}\left(r, g^{\prime}\right)-\bar{Z}_{R}^{S}\left(r, f^{\prime}\right)-\bar{Z}_{R}^{S}\left(r, g^{\prime}\right)
$$

Moreover, in the hypothesis of Theorems II.2.20, since any zero $\alpha$ of $f-a_{i}$ is a zero of certain $g-a_{j}$ with the same multiplicity order, we have $\omega_{\alpha}\left(f^{\prime}\right)=\omega_{\alpha}\left(g^{\prime}\right)$. Consequently,we obtain this improvement of $(3 A)$ :

$$
\sum_{j=1}^{n} \bar{Z}_{R}\left(r, f-a_{j}\right) \leq \bar{Z}_{R}(r, H)+\frac{1}{2}\left[\bar{Z}_{R}\left(r, f^{\prime}\right)+\bar{Z}_{R}\left(r, g^{\prime}\right)-\bar{Z}_{R}^{S}\left(r, f^{\prime}\right)-\bar{Z}_{R}^{S}\left(r, g^{\prime}\right)\right] .
$$


By Corollary II.1.5.1 we know that $\bar{Z}_{R}(r, H) \leq N_{R}(r, H)+O(\log (r)), \quad(r \in J)$, hence

$\sum_{j=1}^{n} \bar{Z}_{R}\left(r, f-a_{j}\right) \leq N_{R}(r, H)+\bar{Z}_{R}\left(r, f^{\prime}\right)+\bar{Z}_{R}\left(r, g^{\prime}\right)-\bar{Z}_{R}^{S}\left(r, f^{\prime}\right)-\bar{Z}_{R}^{S}\left(r, g^{\prime}\right)+O(\log (r))$ $(r \in I)$, and similarly

$\sum_{j=1}^{n} \bar{Z}_{R}\left(r, g-a_{j}\right) \leq N_{R}(r, H)+\bar{Z}_{R}\left(r, f^{\prime}\right)+\bar{Z}_{R}\left(r, g^{\prime}\right)-\bar{Z}_{R}^{S}\left(r, f^{\prime}\right)-\bar{Z}_{R}^{S}\left(r, g^{\prime}\right)+O(\log (r))$ $(r \in I)$, hence

$$
\sum_{j=1}^{n} \bar{Z}_{R}\left(r, f-a_{j}\right)+\bar{Z}_{R}\left(r, g-a_{j}\right)
$$

$(4 A) \leq 2 N_{R}(r, H)+2\left[\bar{Z}_{R}\left(r, f^{\prime}\right)+\bar{Z}_{R}\left(r, g^{\prime}\right)-\bar{Z}_{R}^{S}\left(r, f^{\prime}\right)-\bar{Z}_{R}^{S}\left(r, g^{\prime}\right)\right]+O(\log (r))$.

And in the hypothesis of Theorem II.2.20, by (3B) we obtain

$$
\sum_{j=1}^{n} \bar{Z}_{R}\left(r, f-a_{j}\right)+\bar{Z}_{R}\left(r, g-a_{j}\right)
$$

$(4 B) \leq 2 N_{R}(r, H)+\left[\bar{Z}_{R}\left(r, f^{\prime}\right)+\bar{Z}_{R}\left(r, g^{\prime}\right)-\bar{Z}_{R}^{S}\left(r, f^{\prime}\right)-\bar{Z}_{R}^{S}\left(r, g^{\prime}\right)\right]+O(\log (r))$.

Consider now $N_{R}(r, H)$, and let $\eta$ be a pole of $H$. Either $\eta$ is a zero of $F^{\prime} G^{\prime}$ or it is a pole of $F G$. Let $\zeta$ be the counting function of the poles of $H$ occuring when $F G$ has a pole. So, we have

$$
N(r, H)=\bar{Z}_{R}\left(r, F^{\prime} G^{\prime}\right)+\zeta(r)
$$

Suppose first $\eta$ is a zero of $F^{\prime}$ but is not a pole of $F G$. Either $\eta$ is a zero of $f^{\prime}$, or it is a zero of $P^{\prime}(f)$, or it is a pole of $f$. Consequently, $\eta$ is not a zero of $P(f)$ and we have $\bar{Z}_{R}\left(r, F^{\prime}\right) \leq \bar{Z}_{R}^{S}\left(r, f^{\prime}\right)+\bar{Z}_{R}\left(r, P^{\prime}(f)\right)+\bar{N}_{R}(r, f)$. Since

$$
P^{\prime}(X)=n(n-1)(n-2)(X-1)^{2} X^{n-3},
$$

we have $\bar{Z}_{R}\left(r, P^{\prime}(f)\right) \leq 2 T_{R}(r, f), \quad \bar{Z}_{R}\left(r, P^{\prime}(g)\right) \leq 2 T_{R}(r, g)$, and therefore

$$
\bar{Z}_{R}\left(r, F^{\prime}\right) \leq \bar{Z}_{R}^{S}\left(r, f^{\prime}\right)+2 T(r, f)+\bar{N}_{R}(r, f) .
$$

Similarly, if $\eta$ is a zero of $G^{\prime}$ but is not a pole of $F G$, we have

$$
\bar{Z}_{R}\left(r, G^{\prime}\right) \leq \bar{Z}_{R}^{S}\left(r, g^{\prime}\right)+2 T(r, g)+\bar{N}_{R}(r, g) .
$$

Suppose now $\eta$ is a pole of $F G$. Then $\eta$ is a zero of $P(f)$ and $P(g)$ (we notice that when $\eta$ is a zero of $P(f)$, it is a zero of $P(g)$, and vice-versa), and then by Lemma II.2.17, it may not be a pole of $H$ when it is a zero of same order of $P(f)$ and $P(g)$. Consequently, in the hypothesis of Theorem II.2.19 $\zeta(r)$ satisfies

$$
\zeta(r) \leq \bar{Z}_{R}\left(r, f^{\prime}\right)-\bar{Z}_{R}^{S}\left(r, f^{\prime}\right)+\bar{Z}_{R}\left(r, g^{\prime}\right)-\bar{Z}_{R}^{S}\left(r, g^{\prime}\right),
$$

and therefore by (5), (6) and (7) we obtain

$$
\begin{gathered}
N_{R}(r, H) \leq \bar{Z}_{R}^{S}\left(r, f^{\prime}\right)+\bar{Z}_{R}^{S}\left(r, g^{\prime}\right)+2 T(r, f)+2 T(r, g)+\bar{N}_{R}(r, f)+\bar{N}(r, g) \\
+\bar{Z}_{R}\left(r, f^{\prime}\right)-\bar{Z}_{R}^{S}\left(r, f^{\prime}\right)+\bar{Z}_{R}\left(r, g^{\prime}\right)-\bar{Z}_{0}^{S}\left(r, g^{\prime}\right)
\end{gathered}
$$


hence

$N_{R}(r, H) \leq 2 T(r, f)+2 T(r, g)+\bar{N}_{R}(r, f)+\bar{N}_{R}(r, g)+\bar{Z}_{R}\left(r, f^{\prime}\right)+\bar{Z}_{R}\left(r, g^{\prime}\right)$.

Thus by (4A), in the hypothesis of Theorems II.2.19, we obtain

$$
\sum_{j=1}^{n} \bar{Z}_{R}\left(r, f-a_{j}\right)+\bar{Z}_{R}\left(r, g-a_{j}\right) \leq
$$

$4(T(r, f)+T(r, g))+2\left(\bar{N}_{R}(r, f)+2 \bar{N}_{R}(r, g)\right)+4\left(\bar{Z}_{R}\left(r, f^{\prime}\right)+\bar{Z}_{R}\left(r, g^{\prime}\right)\right)+O(\log (r))$. Now, in the hypotheses of Theorem II.2.20 since the order of a zero is the same for $P(f)$ and $P(g)$, the counting function $\zeta$ is identically 0 , so by, (5) (6), (7) we have

$$
N_{R}(r, H) \leq 2 T_{R}(r, f)+2 T_{R}(r, g)+\bar{N}_{R}(r, f)+\bar{N}(r, g)+\bar{Z}_{R}^{S}\left(r, f^{\prime}\right)+\bar{Z}_{R}^{S}\left(r, g^{\prime}\right),
$$

and therefore by $(4 \mathrm{~B})$ we obtain

$$
\sum_{j=1}^{n} \bar{Z}_{R}\left(r, f-a_{j}\right)+\bar{Z}_{R}\left(r, g-a_{j}\right) \leq 4\left(T_{R}(r, f)+T_{R}(r, g)\right)+2(\bar{N}(r, f)
$$

$(8 B)+2 \bar{N}(r, g))+\left(\bar{Z}_{R}\left(r, f^{\prime}\right)+\bar{Z}_{R}\left(r, g^{\prime}\right)\right)+\left(\bar{Z}_{R}^{S}\left(r, f^{\prime}\right)+\bar{Z}_{R}^{S}\left(r, g^{\prime}\right)\right)+O(\log (r))$.

Now, applying Theorem II.1.15 to $f$ and $g$ at the points $a_{j}(1 \leq j \leq n)$, we have:

$$
\begin{gathered}
(n-1) T_{R}(r, f) \leq \bar{N}(r, f)+\sum_{j=1}^{n} \bar{Z}_{R}\left(r, f-a_{j}\right)-Z_{R}^{S}\left(r, f^{\prime}\right)+O(\log (r))(r \in I), \\
(n-1) T_{R}(r, g) \leq \bar{N}_{R}(r, g)+\sum_{j=1}^{n} \bar{Z}_{R}\left(r, g-a_{j}\right)-Z_{R}^{S}\left(r, g^{\prime}\right)-\log r+O(\log (r)),(r \in I)
\end{gathered}
$$

hence,

$$
\begin{gathered}
(n-1)\left(T_{R}(r, f)+T_{R}(r, g)\right) \leq \bar{N}_{R}(r, f)+\bar{N}_{R}(r, g) \\
+\sum_{j=1}^{n} \bar{Z}_{R}\left(r, f-a_{j}\right)+\bar{Z}_{R}\left(r, g-a_{j}\right)-Z_{R}^{S}\left(r, f^{\prime}\right)-Z_{R}^{S}\left(r, g^{\prime}\right)+O(\log (r))(r \in I) .
\end{gathered}
$$

hence by $(8 \mathrm{~A})$, in the hypotheses of Theorems II.2.19, we obtain

$$
\begin{gathered}
(n-1)\left(T_{R}(r, f)+T_{R}(r, g)\right) \leq 3\left(\bar{N}_{R}(r, f)+\bar{N}(r, g)\right) \\
+4(T(r, f)+T(r, g))+4\left(\bar{Z}_{R}\left(r, f^{\prime}\right)+\bar{Z}_{R}\left(r, g^{\prime}\right)\right)+O(\log (r))(r \in I)
\end{gathered}
$$

So, by Theorem II.1.9 we can derive: $(n-1)\left(T_{R}(r, f)+T_{R}(r, g)\right)$

$$
\leq 3\left(\bar{N}_{R}(r, f)+\bar{N}_{R}(r, g)\right)+4\left(T_{R}(r, f)+T_{R}(r, g)\right)+4\left(Z_{R}(r, f)+Z_{R}(r, g)\right)
$$

$$
+4\left(\bar{N}_{R}(r, f)+\bar{N}_{R}(r, g)\right)+O(\log (r))(r \in I)
$$

(resp. $r \in J$.

Therefore we obtain $n \leq 16$ and if $f, g$ belong to $\mathcal{A}^{*}(D)$, then $n \leq 9$.

Next, by (8B), in the hypotheses of Theorem II.2.20, we obtain

$$
\begin{aligned}
& \quad(n-1)\left(T_{R}(r, f)+T_{R}(r, g)\right) \leq 3\left(\bar{N}_{R}(r, f)+\bar{N}_{R}(r, g)\right)+4\left(T_{R}(r, f)+T_{R}(r, g)\right) \\
& +\bar{Z}_{R}\left(r, f^{\prime}\right)+\bar{Z}_{R}\left(r, g^{\prime}\right)+O(\log (r))(r \in I)(\text { resp. } r \in J)
\end{aligned}
$$

Hence by Theorem II.1.9 we obtain: $(n-1)\left(T_{R}(r, f)+T_{R}(r, g)\right) \leq 3\left(\bar{N}_{R}(r, f)+\bar{N}_{R}(r, g)\right)+4\left(T_{R}(r, f)+T_{R}(r, g)\right)$ 
$+\left(Z_{R}(r, f)+Z_{R}(r, g)\right)+\bar{N}_{R}(r, f)+\bar{N}_{R}(r, g)+O(\log (r))(r \in I)(\operatorname{resp} . \quad r \in J)$ Consequently, $n \leq 10$ in Theorem II.2.20. Moreover, if $f, g$ belong to $\mathcal{A}(\mathbb{I K})$ or to $\mathcal{A}^{*}(D)$, then we obtain $n \leq 6$.

Acknowledgement: We are grateful to the referee for many good remarks on notations.

\section{References}

[1] Bergweiler, W. and Eremenko, A. On the singularities of the inverse to a meromorphic function of finite order, Rev. Mat. Iberoamericana, 11, 355-373 (1995).

[2] Bezivin, J.-P., Boussaf, K., Escassut, A. Zeros of the derivative of a p-adic meromorphic function, Buletin des Sciences Mathématiques 136, 8, 839-847 (2012).

[3] Boussaf, K. Motzkin factorization in algebras of analytic elements, Annales Mathéma-tiques Blaise Pascal 2, n1, p.73-91. (1995).

[4] Boutabaa, A., Théorie de Nevanlinna p-adique, Manuscripta Math. 67, p. 251-269 (1990).

[5] Boutabaa, A. and Escassut, A., URS and URSIMS for p-adic meromorphic functions inside a disk, Proc. of the Edinburgh Mathematical Society 44, p. 485-504 (2001).

[6] Charak, K.S. Value distribution theory of meromorphic functions, Mathematics Newsletter, Vol. 18, no. 4, pp. 1-35 (2009).

[7] Chen, H. and Fang, M. On the value distribution of $f^{n} f^{\prime}$. Science in China, 38 A (7), p. 789-798 (1995).

[8] Cherry, W. and Wang, J. Non-Archimedean Analytic Maps to Algebraic Curves Contemporary Mathematics 303, p. 7-35 (2002).

[9] Escassut, A. Analytic Elements in p-adic Analysis. WSCP Singapore, (1995).

[10] Escassut, A. Ultrametric Banach Algebras. WSCP Singapore, (2003).

[11] Escassut, A. Meromorphic functions of uniqueness. Bulletin des Sciences Mathématiques, Vol 131, issue 3, p. 219-241 (2007).

[12] Escassut, A. Value Distribution in p-adic Analysis. WSCP Singapore, (2015)

[13] Escassut, A. and Ojeda, J. The $p$-adic Hayman conjecture when $n=2$, Complex variable and elliptic equations 59, n.10, p. 1451-1455 (2014).

[14] Frank, G. and Reinders, M. A unique range set for meromorphic functions with 11 elements, Complex Variable Theory Applic. 37, p. 185-193 (1998).

[15] Fujimoto, H. On uniqueness of Meromorphic Functions sharing finite sets, Amer. J. Math. 122 no. 6, p. 1175-1203 (2000).

[16] Ha H. K. On p-adic meromorphic functions, Duke Mathematical Journal, 50, 695-711 (1983)

[17] Hanyak, M.O. and Kondratyuk A.A. Meromorphic functions in m-punctured complex planes, Matematychni Studii 27, 1, p. 53-69 (2007).

[18] Hu, P.C. and Yang, C.C. Meromorphic Functions over non-Archimedean Fields, Kluwer Academic Publishers, (2000).

[19] Krasner, M. Prolongement analytique uniforme et multiforme dans les corps valués complets. Les tendances géométriques en algèbre et théorie des nombres, Clermont-Ferrand, p.94141 (1964). Centre National de la Recherche Scientifique (1966), (Colloques internationaux de C.N.R.S. Paris, 143)

[20] Lazard, M. Les zéros des fonctions analytiques sur un corps valué complet, IHES, Publications Mathématiques no. 14, pp. 47-75 ( 1962).

[21] Min Ru, M. Proceedings of the AMS 129,n. 5, p. 1263-1269 (2001).

[22] Motzkin, E. La décomposition d'un élément analytique en facteurs singuliers, Ann. Inst. Fourier 27, n 1, p. 67-82 (1977).

[23] Nevanlinna, R. Le théorème de Picard-Borel et la théorie des fonctions méromorphes. Gauthiers-Villars, Paris (1929).

[24] Ojeda, J. On Hayman's Conjecture over a p-adic field, Taiwanese Journal of Mathematics 12 (9), p. 2295-2313, (2008).

[25] Yamanoi, K. The second main theorem for small functions and related problems, Acta Mathematica 192, p. 225-294 (2004). 
Université Clermont Auvergnel, Laboratoire de Mathématiques Blaise Pascal, UMR CNRS, BP 10448, 63000 Clermont-Ferrand, France

E-mail address: alain.escassut@math.univ-bpclermont.fr

Institute of Mathematics, Vietnam Academy of Science and Technology 18, Hoang Quoc Viet, Hanoi, Vietnam

and: Institute of Mathematics and Applied Sciences (Timas), Thang Long UniverSity, HanoI, Vietnam

E-mail address: tthan@math.ac.vn 